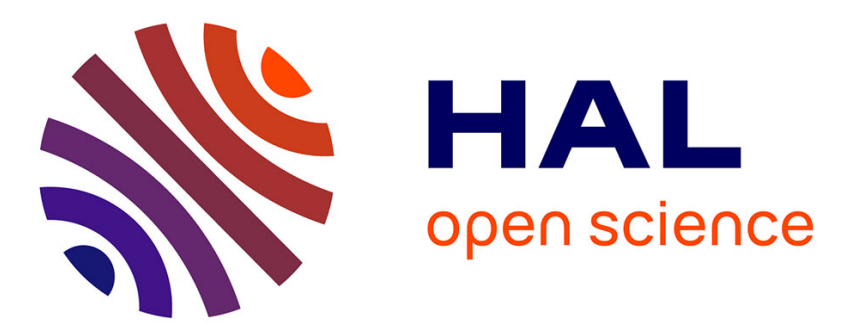

\title{
Canonical lossless state-space systems: Staircase forms and the Schur algorithm
}

Ralf L.M. Peeters, Bernard Hanzon, Martine Olivi

\section{To cite this version:}

Ralf L.M. Peeters, Bernard Hanzon, Martine Olivi. Canonical lossless state-space systems: Staircase forms and the Schur algorithm. Linear Algebra and its Applications, 2007, 425, pp.404-433. 10.1016/j.laa.2006.09.029 . inria-00546790

\section{HAL Id: inria-00546790 \\ https://hal.inria.fr/inria-00546790}

Submitted on 15 Dec 2010

HAL is a multi-disciplinary open access archive for the deposit and dissemination of scientific research documents, whether they are published or not. The documents may come from teaching and research institutions in France or abroad, or from public or private research centers.
L'archive ouverte pluridisciplinaire HAL, est destinée au dépôt et à la diffusion de documents scientifiques de niveau recherche, publiés ou non, émanant des établissements d'enseignement et de recherche français ou étrangers, des laboratoires publics ou privés. 


\title{
Canonical lossless state-space systems: staircase forms and the Schur algorithm
}

\author{
Ralf L.M. Peeters \\ Dept. Mathematics \\ Universiteit Maastricht \\ ralf.peeters@math.unimaas.nl
}

\author{
Bernard Hanzon \\ School of Mathematical Sciences \\ University College Cork \\ b.hanzon@ucc.ie
}

\author{
Martine Olivi \\ Projet APICS \\ INRIA, Sophia Antipolis \\ martine.olivi@sophia.inria.fr
}

\begin{abstract}
A new finite atlas of overlapping balanced canonical forms for multivariate discrete-time lossless systems is presented. The canonical forms have the property that the controllability matrix is positive upper triangular up to a suitable permutation of its columns. This is a generalization of a similar balanced canonical form for continuous-time lossless systems. It is shown that this atlas is in fact a finite sub-atlas of the infinite atlas of overlapping balanced canonical forms for lossless systems that is associated with the tangential Schur algorithm; such canonical forms satisfy certain interpolation conditions on a corresponding sequence of lossless transfer matrices. The connection between these balanced canonical forms for lossless systems and the tangential Schur algorithm for lossless systems is a generalization of the same connection in the SISO case that was noted before. The results are directly applicable to obtain a finite sub-atlas of multivariate input-normal canonical forms for stable linear systems of given fixed order, which is minimal in the sense that no chart can be left out of the atlas without losing the property that the atlas covers the manifold.
\end{abstract}

Keywords: Lossless systems, input normal forms, output normal forms, balanced canonical forms, model reduction, MIMO systems, tangential Schur algorithm.

\section{Introduction}

In linear systems theory there has been a longstanding program in developing balanced realizations, balanced canonical forms and associated parameterizations for stable linear systems and for various other classes of linear systems. The classical Gramian based concept of balancing as introduced by Moore, see [10], applies to stable systems and allows one to develop parameterizations in which system stability is a built-in property. One of the motivations for the interest in balancing is that it leads to a simple method for model order reduction, namely by truncation of (the last entries of) the state vector.

However truncation does not always lead to a minimal system. Therefore there has been research into balanced canonical forms which do have the property that truncation of the last entries in the state vector leads to a minimal system. For continuous-time systems this has led to the original balanced canonical form of Ober (see [11]) and to the new balanced canonical form of Hanzon (see [5]; see also [12]). This last balanced canonical form is based on the idea that if the controllability matrix is positive upper triangular (i.e., the controllability matrix forms an upper triangular matrix with positive entries on the pivot positions), then truncation of the last entries of the state vector leads again to a 
system with a positive upper triangular controllability matrix, hence is controllable. Because this is in the balanced continuous-time case, the controllability property here implies that the resulting system is again minimal and balanced.

To use similar ideas to build overlapping balanced canonical forms is more involved. For continuoustime lossless systems, which form the key to these problems, a generalization of positive upper triangular matrices is used in [6]. The idea used there is that it suffices if a column permutation of the controllability matrix is positive upper triangular. Under certain circumstances there will exist an associated column permutation (we also speak of a shuffle of columns in this context) of the so-called realization matrix, which allows one to proceed with the construction.

In the case of discrete-time systems the situation is somewhat more complicated because it is known that starting from a balanced realization, truncation of the state vector will normally not lead to a balanced state-space system. In the case of SISO lossless discrete-time systems a balanced canonical form with a simple positive upper triangular controllability matrix was presented in [8]. Also the possibilities for model reduction by truncation, combined with a correction of some sort to arrive at a balanced realization of a lossless system, are discussed there.

In the current paper we treat the case of MIMO lossless discrete-time systems. We present overlapping balanced canonical forms which have the property that the corresponding controllability matrix is positive upper triangular, up to a column permutation. In this sense it is close to the corresponding results in [6]; however, here a generalization is presented which simplifies the presentation and which can, as a spin-off, also be used in the continuous-time case. The precise relation with the approach taken in [6] will be made clear. The results on the relation between a specific triangular pivot structure in controllable pairs, which we call "staircase forms", and an associated triangular pivot structure in the controllability matrices are also of interest outside the context of lossless systems.

In [8] a connection was shown between the balanced canonical forms there presented and the Schur algorithm for scalar lossless discrete-time transfer functions. In [7] it is shown how the parameterizations for multivariable rational lossless transfer matrices by Schur parameters, based on the so-called tangential Schur algorithm, can likewise be lifted into parameterizations by Schur parameters of balanced state-space canonical forms of lossless systems. One of the main results of the current paper is to show how the atlas of overlapping balanced canonical forms presented in this paper can be obtained as a finite sub-atlas of the infinite atlas of overlapping balanced canonical forms corresponding to the tangential Schur algorithm. In fact, a certain well-specified choice of so-called direction vectors in the tangential Schur algorithm leads to the balanced canonical forms presented here.

Although a generalization of the results of this paper to the case of complex-valued systems is straightforward, we shall restrict the discussion to the case of real-valued systems only for ease of presentation.

\section{Preliminaries}

\subsection{State space systems and realization theory}

Consider a linear time-invariant state-space system in discrete time with $m$ inputs and $m$ outputs:

$$
\begin{aligned}
& x_{t+1}=A x_{t}+B u_{t}, \\
& y_{t}=C x_{t}+D u_{t},
\end{aligned}
$$

with $t \in \mathbb{Z}, x_{t} \in \mathbb{R}^{n}$ for some nonnegative integer $n$ (the state space dimension), $u_{t} \in \mathbb{R}^{m}$ and $y_{t} \in \mathbb{R}^{m}$. The matrices $A, B, C$ and $D$ with real-valued entries are of compatible sizes: $n \times n$, 
$n \times m, m \times n$ and $m \times m$, respectively. The corresponding transfer matrix of this system is given by $G(z)=D+C\left(z I_{n}-A\right)^{-1} B$, which is an $m \times m$ matrix with rational functions as its entries. The controllability matrix $K$ and the observability matrix $O$ associated with this system are defined as the block-partitioned matrices

$$
K=\left[B, A B, \ldots, A^{n-1} B\right], \quad O=\left[\begin{array}{c}
C \\
C A \\
\vdots \\
C A^{n-1}
\end{array}\right] .
$$

The system (or its input pair $(A, B)$ ) is called controllable if $K$ has full row rank $n$ and the system (or its output pair $(C, A)$ ) is called observable if $O$ has full column rank $n$. Minimality holds iff both controllability and observability hold, which holds iff the McMillan degree of $G(z)$ is equal to $n$.

To any such state-space system we associate the following (square) block-partitioned matrix $R$, which we call the realization matrix:

$$
R=\left[\begin{array}{ll}
D & C \\
B & A
\end{array}\right]
$$

The matrix $R$, its $n \times(m+n)$ sub-matrix $[B, A]$, and the associated $n \times n m$ controllability matrix $K$ will all play an important role in the sequel.

\subsection{Stability and balancing}

Let $(A, B, C, D)$ be some state space realization of a transfer matrix $G(z)$. If the eigenvalues of $A$ all belong to the open unit disk in the complex plane, then the matrix $A$ is called (discrete-time) asymptotically stable, and $(A, B, C, D)$ is an asymptotically stable realization of $G(z)$. (For more details on state-space realization theory, see e.g. [9].)

If $(A, B, C, D)$ is an asymptotically stable realization, then the controllability Gramian $W_{c}$ and the observability Gramian $W_{o}$ are well defined as the exponentially convergent series

$$
\begin{aligned}
& W_{c}=\sum_{k=0}^{\infty} A^{k} B B^{T}\left(A^{T}\right)^{k}, \\
& W_{o}=\sum_{k=0}^{\infty}\left(A^{T}\right)^{k} C^{T} C A^{k} .
\end{aligned}
$$

These Gramians are characterized as the unique (and positive semi-definite) solutions of the respective Lyapunov-Stein equations

$$
\begin{aligned}
& W_{c}-A W_{c} A^{T}=B B^{T}, \\
& W_{o}-A^{T} W_{o} A=C^{T} C .
\end{aligned}
$$

A minimal and asymptotically stable state-space realization $(A, B, C, D)$ of a transfer matrix is called balanced if its controllability and observability Gramians $W_{c}$ and $W_{o}$ are both diagonal and equal. Minimality implies that $W_{c}$ and $W_{o}$ are non-singular, hence positive definite. Any minimal and asymptotically stable realization $(A, B, C, D)$ is similar to a balanced realization, meaning that there exists a nonsingular state space transformation matrix $T$ which makes the realization $\left(T A T^{-1}, T B, C T^{-1}, D\right)$ into a balanced realization. 
A system is called input-normal if $W_{c}=I_{n}$ and it is called output-normal if $W_{o}=I_{n}$. Balanced realizations are directly related to input-normal and output-normal realizations, respectively, by diagonal state space transformations. The property of input-normality (resp. output-normality) is preserved under orthogonal state space transformations.

\subsection{Lossless systems, balanced realizations and the tangential Schur algorithm}

A discrete-time system is called lossless if it is stable and its $m \times m$ transfer matrix $G(z)$ is unitary for all complex $z$ with $|z|=1$. It is well-known (cf., e.g., Proposition 3.2 in [7] and the references given there) that $R=\left[\begin{array}{ll}D & C \\ B & A\end{array}\right]$ is a balanced realization matrix of a lossless system if and only if $R$ is an orthogonal matrix and $A$ is asymptotically stable. It then holds that $W_{c}=W_{o}=I_{n}$. For a further background on lossless systems, see e.g. [3].

In [7] an atlas of overlapping balanced canonical forms for lossless discrete-time systems of order $n$ is presented. Also, a closely related atlas is given for (controllable) input-normal pairs $(A, B)$ by considering the quotient space with respect to the orthogonal group. Each of these balanced canonical forms is then characterized (in the real case) by a fixed sequence of $n$ interpolation points $w_{k} \in \mathbb{R}$, $\left|w_{k}\right|<1, k=1, \ldots, n$, and a fixed sequence of $n$ normalized direction vectors $u_{k} \in \mathbb{R}^{m},\left\|u_{k}\right\|=1$, $k=1, \ldots, n$ (which are not to be confused with the input signal applied to a system). Here we shall consider the case $w_{k}=0, k=1, \ldots, n$, hence each balanced canonical form that we consider is determined entirely by the choice of direction vectors. Each such balanced canonical form for inputnormal pairs $(A, B)$ is then parameterized by a sequence of $n$ Schur vectors $v_{k} \in \mathbb{R}^{m}$, with $\left\|v_{k}\right\|<1$ for all $k=1, \ldots, n$. For lossless systems the parameterization also involves an additional $m \times m$ orthogonal matrix $D_{0}$.

In fact, the realization matrix $R$ in this set-up can be written as an orthogonal matrix product:

$$
R=\Gamma_{n} \Gamma_{n-1} \cdots \Gamma_{1} R_{0} \Delta_{1}^{T} \Delta_{2}^{T} \cdots \Delta_{n}^{T}
$$

where for $k=1, \ldots, n$ :

$$
\begin{aligned}
\Gamma_{k} & =\left[\begin{array}{ccc}
I_{n-k} & 0 & 0 \\
0 & V_{k} & 0 \\
0 & 0 & I_{k-1}
\end{array}\right], \\
\Delta_{k} & =\left[\begin{array}{ccc}
I_{n-k} & 0 & 0 \\
0 & U_{k} & 0 \\
0 & 0 & I_{k-1}
\end{array}\right]
\end{aligned}
$$

with an $(m+1) \times(m+1)$ orthogonal matrix block $V_{k}$ given by

$$
V_{k}=\left[\begin{array}{cc}
v_{k} & I_{m}-\left(1-\sqrt{\left.1-\left\|v_{k}\right\|^{2}\right)} \frac{v_{k} v_{k}^{T}}{\left\|v_{k}\right\|^{2}}\right. \\
\sqrt{1-\left\|v_{k}\right\|^{2}} & -v_{k}^{T}
\end{array}\right],
$$

(for $v_{k}=0$ it holds that $V_{k}=\left[\begin{array}{cc}0 & I_{m} \\ 1 & 0\end{array}\right]$ which makes that $V_{k}$ depends smoothly on the entries of $v_{k}$ ) and an $(m+1) \times(m+1)$ orthogonal matrix block $U_{k}$ given by

$$
U_{k}=\left[\begin{array}{cc}
u_{k} & I_{m}-u_{k} u_{k}^{T} \\
0 & u_{k}^{T}
\end{array}\right]
$$


and furthermore an $(n+m) \times(n+m)$ orthogonal matrix $R_{0}$ given by

$$
R_{0}=\left[\begin{array}{cc}
I_{n} & 0 \\
0 & D_{0}
\end{array}\right]
$$

in which $D_{0}$ is $m \times m$ orthogonal.

The interpolation conditions attain the form $G_{k}\left(w_{k}^{-1}\right) u_{k}=v_{k}$, where $G_{k}(z)$ denotes the transfer function associated with the $k$-th order lossless system for which the right lower $(m+k) \times(m+k)$ sub-matrix of $R_{k}=\Gamma_{k} \Gamma_{k-1} \cdots \Gamma_{1} R_{0} \Delta_{1}^{T} \cdots \Delta_{k-1}^{T} \Delta_{k}^{T}$ is a realization matrix. In the present situation with $w_{k}=0$ it follows that $G_{k}\left(w_{k}^{-1}\right)=G_{k}(\infty)=D_{k}$, so that the interpolation conditions can be written as

$$
D_{k} u_{k}=v_{k}
$$

where $\left(A_{k}, B_{k}, C_{k}, D_{k}\right)$ denotes the corresponding state-space realization of the $k$-th order lossless function $G_{k}(z)$.

Note that here we consider the real case with real direction vectors and real Schur parameter vectors. Note further that $R_{0}, \Gamma_{1}, \ldots, \Gamma_{n}$ and $\Delta_{1}, \ldots, \Delta_{n}$ are all orthogonal matrices. It is important to note that the orthogonal matrix product $\Gamma_{n} \Gamma_{n-1} \cdots \Gamma_{1} R_{0}$ in fact forms a positive $m$-upper Hessenberg matrix, i.e. an $(m+n) \times(m+n)$ matrix of which the $m$-th sub-diagonal has positive entries only and of which the last $n-1$ sub-diagonals are all zero. It also follows that if the direction vectors $u_{1}, \ldots, u_{n}$ are taken to be standard basis vectors, then the matrix product $\Delta_{1}^{T} \Delta_{2}^{T} \cdots \Delta_{n}^{T}$ yields a permutation matrix. Hence in that case the balanced realization matrix $R$ is obtained as a column permutation of an orthogonal positive $m$-upper Hessenberg matrix.

\section{Triangular structures in controllable pairs and their controllability matrices}

It is not difficult to see that if the realization matrix $R$ is positive $m$-upper Hessenberg, then (i) the first $n$ columns of the partitioned $n \times(m+n)$ matrix $[B, A]$ form a positive upper triangular matrix, i.e. an upper triangular matrix with only positive entries on the main diagonal, and (ii) the first $n$ columns of the corresponding controllability matrix $K=\left[B, A B, \ldots, A^{n-1} B\right]$ also form a positive upper triangular matrix. (A matrix with this property is called a simple positive upper triangular matrix.) Therefore the realization is controllable. In the discrete-time lossless case, if $R$ is orthogonal, controllability implies that $A$ is asymptotically stable which in turn implies that the realization is minimal.

A balanced realization of a lossless system is determined up to an arbitrary orthogonal change of basis of the state space. The effect of such a change of basis on the controllability matrix is that it is pre-multiplied with an orthogonal matrix. Now it is well-known that any nonsingular square matrix can be written as the product of an orthogonal matrix and a positive upper triangular matrix in a unique way (in numerical linear algebra this is known as the QR-decomposition). If the first $n$ columns of the controllability matrix are linearly independent then a unique orthogonal state-space isomorphism exists which transforms the first $n$ columns of the controllability matrix into a positive upper triangular matrix. This determines a unique local balanced canonical form for lossless systems. In the SISO case it is in fact a global balanced canonical form and it is presented and investigated in [8].

In the MIMO case, the canonical form does not apply to systems which have a non-generic Kronecker structure. This is why this is a local canonical form. In order to see how the concept of requiring the first $n$ columns of the controllability matrix $K$ to be positive upper triangular can be generalized 
to obtain an atlas of local canonical forms in the MIMO case, we will consider the relation between triangular structures in the partitioned matrix $[B, A]$ and triangular structures in the corresponding controllability matrix $K=\left[B, A B, \ldots, A^{n-1} B\right]$. The following definitions will turn out to be useful.

Definition 3.1 Let $n$ be a fixed positive integer. Consider a vector $v \in \mathbb{R}^{n}$.

(a) The vector $v$ is called a pivot vector with a pivot at position $k$, or a pivot- $k$ vector for short, if $\in\{1, \ldots, n\}$ is an integer for which the entry $v(k)$ is strictly positive and the entries $v(j)$ with $j>k$ are all zero.

(b) The vector $v$ is called a positive pivot- $k$ vector if it is a pivot- $k$ vector for which in addition the entries $v(j)$ with $j<k$ are all strictly positive too.

Definition 3.2 For given positive integers $n$ and $r$, consider a mapping $J:\{1, \ldots, n\} \rightarrow\{0,1, \ldots, r\}$ which is written in short-hand notation as $J=\left\{j_{1}, j_{2}, \ldots, j_{n}\right\}$.

(a) Associated with $J$, the mapping $J^{+}: \mathcal{D}_{J}^{+} \rightarrow \mathcal{R}_{J}^{+}$is defined as the restriction of $J$ to $\mathcal{D}_{J}^{+}$which is the largest subset of $\{1, \ldots, n\}$ on which $J$ is nonzero; the co-domain $\mathcal{R}_{J}^{+}$is the corresponding range of positive values occurring as images under $J$.

(b) The mapping $J$ is called a pivot structure if $J^{+}$is a bijection. Then the inverse of $J^{+}$is denoted by $Q^{+}$and the extended mapping $Q:\{1, \ldots, r\} \rightarrow\{0,1, \ldots, n\}$ is written in short-hand notation as $Q=\left\{q_{1}, q_{2}, \ldots, q_{r}\right\}$ and defined by: $q_{k}=Q^{+}(k)$ for $k \in \mathcal{R}_{J}^{+}$, and $q_{k}=0$ otherwise.

(c) An $n \times r$ matrix $M$ is said to have a pivot structure $J$ if for each $k \in \mathcal{D}_{J}^{+}$it holds that column $j_{k}$ of $M$ is a pivot- $k$ vector. (Equivalently, each column $\ell$ of $M$ is a pivot-q $q_{\ell}$ vector, where 'a pivot-0 vector' is synonymous to 'not a pivot vector'.)

(d) A pivot structure $J$ is called a full pivot structure if $\mathcal{D}_{J}^{+}=\{1, \ldots, n\}$.

Example. Let $n=5$ and $r=8$. Consider the mapping $J:\{1, \ldots, 5\} \rightarrow\{0,1, \ldots, 8\}$ given by $J=\left\{j_{1}, j_{2}, j_{3}, j_{4}, j_{5}\right\}=\{7,1,5,3,6\}$. It follows that the domain and co-domain of $J^{+}$are given by $\mathcal{D}_{J}^{+}=\{1,2,3,4,5\}$ and $\mathcal{R}_{J}^{+}=\{1,3,5,6,7\}$, respectively. Note that $J^{+}$is a bijection, so that $J$ is a pivot structure. Since $\mathcal{D}_{J}^{+}=\{1, \ldots, n\}$ it holds that $J$ defines a full pivot structure. The mapping $Q$, which extends the inverse mapping $Q^{+}$of $J^{+}$, is given by: $Q=\left\{q_{1}, q_{2}, q_{3}, q_{4}, q_{5}, q_{6}, q_{7}, q_{8}\right\}=$ $\{2,0,4,0,3,5,1,0\}$. Any $5 \times 8$ matrix $M$ which has the full pivot structure $J$ is of the following form:

$$
M=\left[\begin{array}{cccccccc}
* & * & * & * & * & * & + & * \\
+ & * & * & * & * & * & 0 & * \\
0 & * & * & * & + & * & 0 & * \\
0 & * & + & * & 0 & * & 0 & * \\
0 & * & 0 & * & 0 & + & 0 & *
\end{array}\right]
$$

where the entries denoted by $*$ are allowed to have an arbitrary value and the entries denoted by + are required to be (strictly) positive. Note that $J$ addresses the entries denoted by + for each row and $Q$ specifies the same entries for each column.

The construction of $Q$ from a given pivot structure $J$ induces a mapping $T_{n, r}: J \mapsto Q$. From the fact that $J^{+}$and $Q^{+}$are each others inverse, is not difficult to see that $T_{r, n}$ provides the inverse of $T_{n, r}$. The sets $\left\{\left(k, j_{k}\right) \mid k \in \mathcal{D}_{J}^{+}\right\}$and $\left\{\left(q_{\ell}, \ell\right) \mid \ell \in \mathcal{R}_{J}^{+}\right\}$obviously coincide: the mappings $J$ and $Q$ both serve to specify the same set of pivot locations for an $n \times r$ matrix, satisfying the rule that in each row and in each column of that matrix at most one pivot location is selected. The mapping $J$ specifies these pivot locations in a row-oriented fashion, the mapping $Q$ in a column-oriented fashion. 
For a full pivot structure it holds that $n \leq r$. If $J=\left\{j_{1}, j_{2}, \ldots, j_{n}\right\}$ is a full pivot structure for an $n \times r$ matrix $M$, then the ordered selection of columns $j_{1}, j_{2}, \ldots, j_{n}$ from $M$ constitutes a positive upper triangular $n \times n$ sub-matrix. In this way, positive upper triangularity is generalized by the concept of a full pivot structure.

As explained before, if a block-partitioned $n \times(m+n)$ matrix $[B, A]$ is simple positive upper triangular (i.e., it has the full pivot structure $J$ with $j_{k}=k$ for $k=1, \ldots, n$ ) then the associated (finite or infinite) controllability matrix $K=\left[B, A B, A^{2} B, \ldots\right]$ also is simple positive upper triangular. We now proceed to investigate the question which full pivot structures for $[B, A]$ induce full pivot structures for $K$. Conversely, it is of interest to determine which full pivot structures for $K$ are induced by full pivot structures for $[B, A]$. The latter question is more involved and it is studied in detail in the following section. Here we address the former question for which the following definition is useful.

Definition 3.3 Let $m$ and $n$ be given positive integers.

(a) A pivot structure $F$ for an $n \times n$ matrix $A$ is called a staircase form for $A$ if $F^{+}$is monotonically increasing having the range $\mathcal{R}_{F}^{+}=\left\{1,2, \ldots, p_{A}\right\}$. Here $p_{A}$ denotes the number of pivots, i.e. the number of elements in $\mathcal{D}_{F}^{+}$.

(b) A pivot structure $J=\left\{j_{1}, \ldots, j_{n}\right\}$ for an $n \times(m+n)$ block-partitioned matrix $[B, A]$ induces a pivot structure $P=\left\{p_{1}, \ldots, p_{n}\right\}$ for the matrix $A$ as given by $p_{k}=\max \left\{j_{k}-m, 0\right\}$ for $k=1, \ldots, n$. (c) A full pivot structure $J=\left\{j_{1}, j_{2}, \ldots, j_{n}\right\}$ for an $n \times(m+n)$ block-partitioned matrix $[B, A]$ is called an admissible pivot structure for $[B, A]$ if it holds that: (i) $B$ has a pivot-1 vector, i.e. $1 \leq j_{1} \leq$ $m$, and (ii) the pivot structure $P$ induced by $J$ constitutes a staircase form for $A$.

Of course, a pivot structure $J=\left\{j_{1}, \ldots, j_{n}\right\}$ for an $n \times(m+n)$ block-partitioned matrix $[B, A]$ also induces a pivot structure for the matrix $B$. For several purposes, the induced pivot structures for $A$ and $B$ are more conveniently described in terms of the associated column-oriented description $Q=\left\{q_{1}, \ldots, q_{m+n}\right\}$ for $[B, A]$. For the matrix $A$ it holds that the associated column-oriented pivot structure $S=\left\{s_{1}, \ldots, s_{n}\right\}$ satisfies $s_{k}=q_{m+k}$ for all $k=1, \ldots, n$. For the matrix $B$ the associated column-oriented pivot structure is the restriction of $Q$ to the domain $\{1, \ldots, m\}$, simply described by the sequence $\left\{q_{1}, \ldots, q_{m}\right\}$.

Example. Let $m=4, n=6$ and consider the full pivot structure $J=\{3,1,5,6,4,7\}$ for the $n \times(m+n)$ partitioned matrix $[B, A]$. The corresponding column-oriented description is given by $Q=\{2,0,1,5,3,4,6,0,0,0\}$. The matrix $[B, A]$ therefore has the form:

$$
[B, A]=\left[\begin{array}{cccc|cccccc}
* & * & + & * & * & * & * & * & * & * \\
+ & * & 0 & * & * & * & * & * & * & * \\
0 & * & 0 & * & + & * & * & * & * & * \\
0 & * & 0 & * & 0 & + & * & * & * & * \\
0 & * & 0 & + & 0 & 0 & * & * & * & * \\
0 & * & 0 & 0 & 0 & 0 & + & * & * & *
\end{array}\right]
$$

The induced pivot structure for the matrix $A$ is given by $P=\left\{p_{1}, p_{2}, p_{3}, p_{4}, p_{5}, p_{6}\right\}=\{0,0,1,2,0,3\}$, which follows from $p_{k}=\max \left\{j_{k}-4,0\right\}$ for $k=1,2, \ldots, 6$. The associated column-oriented description is then given by $S=\left\{s_{1}, s_{2}, s_{3}, s_{4}, s_{5}, s_{6}\right\}=\{3,4,6,0,0,0\}=\left\{q_{5}, q_{6}, q_{7}, q_{8}, q_{9}, q_{10}\right\}$. The function $P^{+}$is given by the pairs $(3,1),(4,2)$ and $(6,3)$; the inverse $S^{+}$is given by $(1,3),(2,4)$ and $(3,6)$. Clearly, $P^{+}$is monotonically strictly increasing (and equivalently $S^{+}$is monotonically strictly increasing) so that $P$ is a staircase form for $A$. This is clearly illustrated by the pattern constituted by the entries denoted by + in the matrix $A$ above. Also, the matrix $B$ has a pivot- 1 column 
as its third column. Therefore, $J$ constitutes an admissible pivot structure for $[B, A]$. Note that the column-oriented description of the pivot structure for the matrix $B$ follows from the restriction of $Q$ as: $\left\{q_{1}, q_{2}, q_{3}, q_{4}\right\}=\{2,0,1,5\}$.

Note that an admissible pivot structure $J$ for $[B, A]$ is totally determined by the induced pivot structure for $B$. In that case, the pivot structure $S$ for the matrix $A$ having a staircase form is given by $\left\{s_{1}, \ldots, s_{n}\right\}=\left\{q_{m+1}, \ldots, q_{m+p_{A}}, 0, \ldots, 0\right\}$ where the subsequence $\left\{q_{m+1}, \ldots, q_{m+p_{A}}\right\}$ is positive and monotonically increasing, consisting of the elements of $\{1,2, \ldots, n\}$ not occurring in $\left\{q_{1}, \ldots, q_{m}\right\}$. For admissibility, the only condition on the column-oriented pivot structure $\left\{q_{1}, \ldots, q_{m}\right\}$ for $B$ is that 1 occurs in this sequence.

If $v$ is a pivot- $k$ vector and $J$ is admissible, then the staircase structure of $A$ implies that $w=A v$ is a pivot- $s_{k}$ vector. For this reason, the function $S$ will be called the successor function. (For convenience we also define $S(0)=0$ and we recall that the terminology 'a pivot-0 vector' is synonymous to 'not a pivot vector'.) The sequence of pivot positions for the vectors $v, A v, A^{2} v, A^{3} v, \ldots$ is then given by $k, S(k), S^{2}(k), S^{3}(k), \ldots$. Conversely, the induced pivot structure $P$ for $A$ is called the predecessor function (here we also introduce $P(0)=0$ ). Recall that $S^{+}$and $P^{+}$are each others inverse.

We have the following result.

Theorem 3.4 Let $m$ and $n$ be given positive integers.

(a) If $J$ is an admissible pivot structure for an $n \times(m+n)$ block-partitioned matrix $[B, A]$, then $K=\left[B, A B, A^{2} B, \ldots\right]$ has a full pivot structure $\widetilde{J}$.

(b) For every non-admissible full pivot structure $J$ there exists an $n \times(m+n)$ matrix $[B, A]$ having the full pivot structure $J$, for which $K=\left[B, A B, A^{2} B, \ldots\right]$ does not have a full pivot structure.

Proof. (a) Admissibility of $J$ implies that $B$ has a pivot- 1 column. Thus, the (infinite) controllability matrix $K$ also has a pivot-1 column, because $B$ is a sub-matrix of $K$. Now consider the induction hypothesis that the controllability matrix is known to have pivots at positions $1,2, \ldots, k$, with $1 \leq k<$ $n$. From the admissible pivot structure of $[B, A]$, either $B$ or $A$ has a pivot- $(k+1)$ column, depending on the value of $j_{k+1}$. If $j_{k+1} \leq m$, then this column is in $B$ hence it also appears in $K$. Otherwise, column $j_{k+1}$ of $[B, A]$ is in fact column $p_{k+1}=j_{k+1}-m$ of $A$. Equivalently, $s_{\ell}=q_{m+\ell}=k+1$ for $\ell=p_{k+1}$. Because of the staircase structure of $A$ (and because the prescribed pivot- 1 column is in $B$ ) it holds that $\ell \leq k$. Since $K$ has a pivot- $\ell$ column according to the induction hypothesis, the matrix product $A K$ now has a pivot- $(k+1)$ column because of the staircase structure of $A$. But $A K$ is a sub-matrix of $K$, whence it follows that $K$ has a pivot- $(k+1)$ column. This shows the induction step. Hence the controllability matrix has a full pivot structure.

(b) See Appendix A.

\section{Remarks.}

(i) For an admissible pivot structure $J$ for $[B, A]$ there is a uniquely determined full pivot structure $\widetilde{J}$ which applies to every controllability matrix $K$ that may occur for each arbitrary matrix $[B, A]$ having the structure $J$. One can easily calculate $\widetilde{J}$ using the numbered Young diagram technique described in the following section. It is most clearly displayed in $K$ for the example where each pivot- $k$ column in $[B, A]$ is set equal to $e_{k}$ and each non-pivot column is set to zero.

(ii) For given $m$ and $n$, the total number of different admissible full pivot structures can be computed from the fact that an admissible pivot structure is completely determined by the pivot structure for $B$. It is given by: $\sum_{\ell=1}^{\min \{m, n\}} \ell !\left(\begin{array}{c}m \\ \ell\end{array}\right)\left(\begin{array}{c}n-1 \\ \ell-1\end{array}\right)$. 


\section{The Young diagram associated with an admissible pivot structure}

Starting from an admissible pivot structure $J$ for $[B, A]$ we now want to analyze the full pivot structure $\widetilde{J}$ for the (finite) controllability matrix $K=\left[B, A B, \ldots, A^{n-1} B\right]$ induced by $J$ and describe their relation.

Admissibility of $J$ implies that $1 \leq j_{1} \leq m$, so that $s_{1}$ is either zero (in which case $A$ has no pivots) or $s_{1}>1$. Together with the staircase form of $A$ this means that for all $k=1, \ldots, n$ either $s_{k}>k$ or $s_{k}=0$. The sequence $\left\{k, S(k), S^{2}(k), S^{3}(k), \ldots\right\}$ therefore is strictly monotonically increasing until at some point the value 0 occurs after which the sequence remains zero. This happens when $S^{t}(k)$ attains a value in $\left\{p_{A}+1, \ldots, n\right\}$. Conversely, starting from a value $\ell>0$ the sequence $\left\{\ell, P(\ell), P^{2}(\ell), P^{3}(\ell), \ldots\right\}$ is strictly monotonically decreasing until at some point the value 0 occurs after which the sequence remains zero. This happens when $P^{t}(\ell)$ attains a value in $\left\{q_{1}, \ldots, q_{m}\right\}$.

In this way, an admissible pivot structure $J$ for $[B, A]$ generates a uniquely specified full pivot structure $\widetilde{J}$ for the controllability matrix $K$. To visualize this, it is helpful to introduce an $m \times n$ array $Y=\left(y_{i, j}\right)$, defined as follows: entry $y_{i, j}$ denotes the pivot position of vector $i$ in the $j$-th block $A^{j-1} B$ of $K$ (so that $\widetilde{J}(k)=(j-1) m+i$ where $i$ and $j$ are such that $y_{i, j}=k$ ). In terms of the column-oriented description $\widetilde{Q}=\left\{\widetilde{q}_{1}, \widetilde{q}_{2}, \ldots, \widetilde{q}_{n m}\right\}$ of the pivot structure of $K$ associated with the row-oriented full pivot structure $\widetilde{J}$, it simply holds that $y_{i, j}=\widetilde{q}_{(j-1) m+i}$ for all $i=1, \ldots, m$ and $j=1, \ldots, n$. The array $Y$ can therefore be regarded as an $m \times n$ matrix representation of $\widetilde{Q}$ which allows a clearer expression of the role played by the block-partitioning of $K$. Obviously, there is a one-to-one correspondence between such an array $Y$ (with entries in $\{0,1, \ldots, n\}$ ) and the function $\widetilde{Q}$ (from $\{1, \ldots, n m\}$ to $\{0,1, \ldots, n\}$ ).

Example. Let $m=4, n=6$ and consider the admissible full pivot structure $J=\{3,1,5,6,4,7\}$ and its associated column-oriented description $Q=\{2,0,1,5,3,4,6,0,0,0\}$ for the $6 \times 10$ partitioned matrix $[B, A]$ given by:

$$
[B, A]=\left[\begin{array}{cccc|cccccc}
* & * & + & * & * & * & * & * & * & * \\
+ & * & 0 & * & * & * & * & * & * & * \\
0 & * & 0 & * & + & * & * & * & * & * \\
0 & * & 0 & * & 0 & + & * & * & * & * \\
0 & * & 0 & + & 0 & 0 & * & * & * & * \\
0 & * & 0 & 0 & 0 & 0 & + & * & * & *
\end{array}\right]
$$

Then the successor function $S$ is given by $S(0)=0$ and $\left\{s_{1}, s_{2}, s_{3}, s_{4}, s_{5}, s_{6}\right\}=\{3,4,6,0,0,0\}$ and the predecessor function $P$ is given by $P(0)=0$ and $\left\{p_{1}, p_{2}, p_{3}, p_{4}, p_{5}, p_{6}\right\}=\{0,0,1,2,0,3\}$. Note that the matrix $K=\left[B, A B, A^{2} B, \ldots\right]$ is of the form:

$$
K=\left[\begin{array}{cccc|cccc|cccc|cc}
* & * & + & * & * & * & * & * & * & * & * & * & \ldots & \ldots \\
+ & * & 0 & * & * & * & * & * & * & * & * & * & \ldots & \ldots \\
0 & * & 0 & * & * & * & + & * & * & * & * & * & \ldots & \ldots \\
0 & * & 0 & * & + & * & 0 & * & * & * & * & * & \ldots & \ldots \\
0 & * & 0 & + & 0 & * & 0 & * & * & * & * & * & \ldots & \ldots \\
0 & * & 0 & 0 & 0 & * & 0 & * & * & * & + & * & \ldots & \ldots
\end{array}\right]
$$

This shows that the induced full pivot structure $\widetilde{J}$ for $K$ is given by $\widetilde{J}=\{3,1,7,5,4,11\}$ and it has an associated column-oriented description $\widetilde{Q}=\{2,0,1,5,4,0,3,0,0,0,6,0, \ldots\}$. The corresponding 
$4 \times 6$ array $Y$ is filled with the values in $\widetilde{Q}$ column after column, yielding the diagram:

$$
Y=\begin{array}{|l|l|l|l|l|l|}
\hline 2 & 4 & 0 & 0 & 0 & 0 \\
\hline 0 & 0 & 0 & 0 & 0 & 0 \\
\hline 1 & 3 & 6 & 0 & 0 & 0 \\
\hline 5 & 0 & 0 & 0 & 0 & 0 \\
\hline
\end{array}
$$

Note that the first column of $Y$ specifies the pivot structure of $B$, i.e.: $\left\{q_{1}, q_{2}, q_{3}, q_{4}\right\}=\{2,0,1,5\}$. The other entries of $Y$ satisfy the rule $y_{i, j+1}=S\left(y_{i, j}\right)$.

Theorem 4.1 Let $J$ be an admissible full pivot structure for the block-partitioned matrix $[B, A]$, with an associated column-oriented description $Q=\left\{q_{1}, \ldots, q_{m+n}\right\}$ and the successor function $S$ given by $S=\left\{s_{1}, \ldots, s_{n}\right\}=\left\{q_{m+1}, \ldots, q_{m+n}\right\}$ and $S(0)=0$. Then $J$ induces a full pivot structure $\widetilde{J}$ for the (finite) controllability matrix $K=\left[B, A B, \ldots, A^{n-1} B\right]$ which is specified in terms of the $m \times n$ array $Y$ associated with $\widetilde{Q}$ as follows:

(i) $y_{i, 1}=q_{i}$ for $i=1, \ldots, m$;

(ii) $y_{i, j+1}=S\left(y_{i, j}\right)$ for $i=1, \ldots, m$ and $j=1, \ldots, n-1$.

Proof. As argued in the previous section, the admissible pivot structure $J$ for $[B, A]$ is entirely determined by the induced column-oriented pivot structure $\left\{q_{1}, \ldots, q_{m}\right\}$ for $B$. Given these (prescribed) pivot positions for the columns of $B$, the resulting pivot positions for the columns of the block $A B$ are given by $\left\{S\left(q_{1}\right), \ldots, S\left(q_{m}\right)\right\}$. Likewise, the pivot positions for the columns of the block $A^{2} B$ are given by $\left\{S^{2}\left(q_{1}\right), \ldots, S^{2}\left(q_{m}\right)\right\}$. Proceeding in this fashion, it follows that the pivot structure $\widetilde{J}$ for $K$ induced by $J$ corresponds to an array $Y$ which is described by: (i) the first column of $Y$, which corresponds to $B$ and satisfies $y_{i, 1}=q_{i}$ for $i=1, \ldots, m$; (ii) the other columns of $Y$, which are given by the recursion $y_{i, j+1}=S\left(y_{i, j}\right)$ for $i=1, \ldots, m$ and $j=1, \ldots, n-1$. In part (a) of the proof of Theorem 3.4 it has already been argued that $\widetilde{J}$ obtained in this way describes a full pivot structure for $K$.

The array $Y$ in the theorem above has the property that the values $1,2, \ldots, n$ all occur precisely once while the other $(m-1) n$ entries are all zero. The set of arrays $Y$ with this property is denoted by $\mathcal{Y}(m, n)$. Clearly, there is a one-to-one correspondence between this set of arrays and the set of full pivot structures for finite controllability matrices $K$ of size $n \times n m$. However, not all the arrays $Y$ in the set $\mathcal{Y}(m, n)$ are induced by some admissible pivot structure $J$ for $[B, A]$. The following definition serves the goal of characterizing the subset of $\mathcal{Y}(m, n)$ of arrays $Y$ that are induced by admissible pivot structures.

Definition 4.2 An array $Y \in \mathcal{Y}(m, n)$ is called an admissible numbered Young diagram if it has the following three properties:

(i) for all $i=1, \ldots, m$ and $j=1, \ldots, n-1$ it holds that $y_{i, j+1}>0$ implies $y_{i, j}>0$;

(ii) the values $n-p_{B}+1, \ldots, n$ all occur in different rows of $Y$ as their last nonzero entries, where $p_{B}$ is the number of nonzero rows of $Y$;

(iii) for all $i, i^{\prime}=1, \ldots, m$ and $j, j^{\prime}=1, \ldots, n-1$ it holds that $y_{i, j+1}>y_{i^{\prime}, j^{\prime}+1}>0$ implies $y_{i, j}>y_{i^{\prime}, j^{\prime}}>0$.

Note that the number of nonzero rows of the array $Y$ corresponding to the induced full pivot structure $\widetilde{J}$ in Theorem 4.1 is equal to the number of nonzero entries in the first column of $Y$, which is equal to the number of pivots in the matrix $B$. This explains the notation $p_{B}$ in the definition above. The 
terminology 'numbered Young diagram' will become more clear below, when the relationship with nice selections and Young diagrams is explained.

Theorem 4.3 (a) Let $J$ be an admissible full pivot structure for the block-partitioned matrix $[B, A]$, then the induced full pivot structure $\widetilde{J}$ for the controllability matrix $K=\left[B, A B, \ldots, A^{n-1} B\right]$ corresponds to an admissible numbered Young diagram $Y$.

(b) Let $Y$ be an admissible numbered Young diagram. Then there exists an admissible full pivot structure $J$ for $[B, A]$ which induces the full pivot structure $\widetilde{J}$ for $K=\left[B, A B, \ldots, A^{n-1} B\right]$ which corresponds to $Y$.

Proof. (a) From Theorem 4.1 we have that $J$ induces the full pivot structure $\widetilde{J}$ for $K$ which corresponds to an array $Y \in \mathcal{Y}(m, n)$ given by: (i) $y_{i, 1}=q_{i}$ for $i=1, \ldots, m$; (ii) $y_{i, j+1}=S\left(y_{i, j}\right)$ for $i=1, \ldots, m$ and $j=1, \ldots, n-1$. Clearly, the $i$-th row of $Y$ is entirely zero if and only if $q_{i}=0$. Hence the number of nonzero rows of $Y$ is equal to the number $p_{B}$ of (prescribed) pivots in $B$. As we have seen, admissibility of $J$ implies that $s_{k}=0$ if and only if $k \in\left\{p_{A}+1, \ldots, p_{A}+p_{B}=n\right\}$. This shows that the last nonzero entries in the $p_{B}$ nonzero rows of $Y$ have the values $n-p_{B}+1, \ldots, n$ and they necessarily all occur in different rows. Next, if $y_{i, j+1}>0$, then $y_{i, j+1}=S\left(y_{i, j}\right)$ with $y_{i, j}>0$ because $S(0)=0$. This relationship is also described by the predecessor function $P$ as $y_{i, j}=P\left(y_{i, j+1}\right)>0$. Note that in fact the restricted positive functions $S^{+}$and $P^{+}$describe this relationship and they are both strictly monotonically increasing because of the staircase property of $A$. Therefore, by application of $P^{+}$, the relationship $y_{i, j+1}>y_{i^{\prime}, j^{\prime}+1}>0$ implies that $y_{i, j}>y_{i^{\prime}, j^{\prime}}>0$. This shows that $Y$ is an admissible numbered Young diagram.

(b) Suppose that $Y \in \mathcal{Y}(m, n)$ is an admissible numbered Young diagram. Consider the $p_{B}$ nonzero rows of $Y$. According to property (ii), the last nonzero entries of these rows precisely cover the range $\left\{n-p_{B}+1, \ldots, n\right\}$. It follows that all the other entries of $Y$ are $\leq n-p_{B}$ because every positive value from $\{1, \ldots, n\}$ occurs exactly once. Now consider the function $S:\{0,1, \ldots, n\} \rightarrow\{0,1, \ldots, n\}$ defined from the values in $Y$ as follows: $S(0)=0, S\left(y_{i, j}\right)=y_{i, j+1}$ for all $i=1, \ldots, m$ and $j=1, \ldots, n-1$, and $S\left(y_{i, n}\right)=0$ for all $i=1, \ldots, m$. Note that the pattern of positive values in the array $Y$ is left-aligned according to property (i). This makes that the definition $S(0)=0$ is consistent with the prescription $S\left(y_{i, j}\right)=y_{i, j+1}$ in situations where $y_{i, j}=0$, and also with the prescription $S\left(y_{i, n}\right)=0$ in situations where $y_{i, n}=0$. Note also that $S(k)>0$ for all $k=1, \ldots, n-p_{B}$ and $S(k)=0$ for $k=n-p_{B}+1, \ldots, n$ (as well as for $k=0$ ). The associated function $S^{+}$is a bijection with domain $\left\{1, \ldots, n-p_{B}\right\}$.

Property (iii) of $Y$ now implies that $S^{+}$is monotonically increasing. To see this, choose positive integers $k$ and $\ell$ with $S(k)>S(\ell)>0$. Then choose the unique integers $i, i^{\prime}, j$ and $j^{\prime}$ such that $y_{i, j+1}=S(k)$ and $y_{i^{\prime}, j^{\prime}+1}=S(\ell)$ and invoke property (iii) to obtain that $k>\ell>0$. Consequently, $S^{+}$can be used to prescribe a staircase form for the matrix $A$. The $p_{B}$ positive values in $\{1, \ldots, n\}$ not occurring in the range of $S^{+}$are precisely those occurring in the first column of $Y$. This first column of $Y$ serves to describe a pivot structure for $B$. Together with $S^{+}$this determines a full pivot structure $J$ for $[B, A]$ in which $A$ has a staircase form. For $J$ to be admissible, it remains to be shown that $B$ has a prescribed pivot- 1 column, or equivalently that one of the entries in the first column of $Y$ is equal to 1. To see this, suppose that for some $y_{i, j}>0$ it holds that $S\left(y_{i, j}\right)=y_{i, j+1}=1$. Then the bijection $S^{+}$can only be monotonically increasing if $S(1)=0$ so that 1 does not belong to the domain of $S^{+}$, which requires 1 to belong to the set of $p_{B}$ largest values $\left\{n-p_{B}+1, \ldots, n\right\}$. But then $y_{i, j}>1$ also belongs to this set and occurs in a different row of $Y$, producing a contradiction.

We thus have established a bijection between admissible pivot structures $J$ for $[B, A]$ and admissible 
numbered Young diagrams $Y$ associated with $K$. To relate these results to the well-known theory of nice selections and dynamical indices, the following definition is useful.

Definition 4.4 Let $m$ and $n$ be given positive integers.

(a) The set $\mathcal{D}(m, n)$ is defined as the set of all multi-indices $d=\left(d_{1}, d_{2}, \ldots, d_{m}\right) \in \mathbb{N}_{0}^{m}$ for which $d_{1}+d_{2}+\ldots+d_{m}=n$.

(b) A selection of $n$ columns from an $n \times n m$ controllability matrix $K=\left[B, A B, \ldots, A^{n-1} B\right]$ is called a nice selection if there exists a multi-index $d \in \mathcal{D}(m, n)$ for which the selected set of columns is given by $\left\{A^{j-1} B e_{i} \mid j \in\left\{1,2, \ldots, d_{i}\right\}\right.$ for $\left.i=1,2, \ldots, m\right\}$.

(c) A nice pivot structure $\widetilde{J}$ for $K$ is a full pivot structure for $K$ which constitutes a nice selection of columns from $K$.

(d) If $\widetilde{J}$ is a nice pivot structure for $K$, then the associated multi-index $d \in \mathcal{D}(m, n)$ is called the vector of dynamical indices and each number $d_{i}$ is called the $i$-th dynamical index $(i=1,2, \ldots, m)$ of the nice pivot structure, or of the input pair $(A, B)$.

Nice selections and vectors of dynamical indices $d$ are useful and well-known concepts for studying the rank structures that can be exhibited by a controllability matrix $K$. The most well-known nice selection is the Kronecker nice selection, which consists of the first $n$ linearly independent columns of $K$. Every nice selection may occur as the Kronecker nice selection for some controllability matrix $K$. (Cf., e.g., [4] and the references given there.) In the concept of nice selections though, there are no a priori rank or linear independence requirements and no triangularity conditions. Conversely, for a nice pivot structure it is not required that the column selection is a Kronecker nice selection. Note also that there are $n$ ! different nice pivot structures all corresponding to the same nice selection.

Above it has been shown that an admissible pivot structure for $[B, A]$ induces a corresponding full pivot structure for $K$ for which the associated array $Y \in \mathcal{Y}(m, n)$ is an admissible numbered Young diagram. Conversely, all admissible numbered Young diagrams are induced in this way. An admissible numbered Young diagram specifies a selection of $n$ columns of $K$, which constitutes an upper triangular sub-matrix; therefore these $n$ columns are linearly independent. From the definition of a nice selection it should be clear that any nice selection can be represented by an $m \times n$ binary array $Z=\left(z_{i, j}\right)$ in the following way: $z_{i, j}=1$ if column $i$ of the $j$-th block $A^{j-1} B$ of $K$ is included in the nice selection, and $z_{i, j}=0$ otherwise. The nonzero entries in such an array $Z$ exhibit a left-aligned pattern and the dynamical index $d_{i}$ denotes the number of nonzero entries in the $i$-th row of $Z$, while $d_{1}+\ldots+d_{m}=n$. Such an array $Z$ is closely related to the concept of a Young diagram, see [2]. As we have seen, any admissible numbered Young diagram $Y$ is left-aligned and it therefore gives rise to an associated nice selection; the induced full pivot structure $\widetilde{J}$ is a nice pivot structure for $K$. This also explains our terminology. For the purpose of the design of local canonical forms for various classes of linear multivariable systems, it is important that there exists an admissible numbered Young diagram for every nice selection. We therefore continue to study the relationship between nice selections and admissible numbered Young diagrams.

Let $Z$ be a Young diagram, i.e., a left-aligned $m \times n$ binary array corresponding to a nice selection with an associated vector of dynamical indices $d=\left(d_{1}, \ldots, d_{m}\right)$. A numbered Young diagram is obtained from $Z$ by replacing the unit entries in $Z$ by the numbers $1,2, \ldots, n$ in some arbitrary order, so that they all occur exactly once. The set of $m \times n$ numbered Young diagrams is the subset of $\mathcal{Y}(m, n)$ of left-aligned arrays. We will now show that for every Young diagram $Z$ there exists an associated admissible numbered Young diagram $Y$. More precisely, we will characterize all the admissible numbered Young diagrams $Y$ that correspond to $Z$. 
To do this, it is convenient to associate with every left-aligned array $Y \in \mathcal{Y}(m, n)$ a corresponding right-aligned array $Y_{r}$ as follows. If $Y$ is left-aligned then this means that there is an associated vector of dynamical indices $d=\left(d_{1}, \ldots, d_{m}\right)$ such that $y_{i j}>0$ iff $j \leq d_{i}$. Thus, the $i$-th row of $Y$ has positive entries at its first $d_{i}$ positions and zero entries at the remaining $n-d_{i}$ positions. Then $Y_{r}$ is defined by: $\left(Y_{r}\right)_{i j}=0$ for $1 \leq j \leq n-d_{i}$ and $\left(Y_{r}\right)_{i j}=y_{i, j-n+d_{i}}$ for $n-d_{i}+1 \leq j \leq n$. In other words: the $d_{i}$ positive entries in the $i$-th row are all shifted $n-d_{i}$ positions to the right.

Proposition 4.5 Let $Z$ be an $m \times n$ Young diagram corresponding to a nice selection with an associated vector of dynamical indices $d=\left(d_{1}, \ldots, d_{m}\right)$. An $m \times n$ left-aligned array $Y$ corresponding to the same vector of dynamical indices $d$, is an admissible numbered Young diagram if and only if there exists an $m \times m$ permutation matrix $\Pi$ for the associated right-aligned array $Y_{r}$ such that the $n$ m-vector vec $\left(\Pi Y_{r}\right)=\left(\left(\Pi Y_{r} e_{1}\right)^{T},\left(\Pi Y_{r} e_{2}\right)^{T}, \ldots,\left(\Pi Y_{r} e_{n}\right)^{T}\right)^{T} \in \mathbb{R}^{n m}$ obtained by stacking the $n$ columns of the array $\Pi Y_{r}$, has the property that if the zero entries are deleted then the $n$-vector $(1,2,3, \ldots, n)^{T}$ is obtained.

Proof. Suppose $Y$ is an admissible numbered Young diagram corresponding to the vector of dynamical indices $d$. Consider the $p_{B}=n-p_{A}$ nonzero values in the last column of $Y_{r}$ (where $p_{B}$ denotes the number of pivots in $B$, i.e. the number of nonzero entries in $\left\{q_{1}, \ldots, q_{m}\right\}$ which also is the number of nonzero rows in $Y$ as well as in $Y_{r}$ ). These values constitute a permutation of the set of values $\left\{p_{A}+1, \ldots, p_{A}+p_{B}=n\right\}$. Now consider the predecessors $\left\{P\left(p_{A}+1\right), \ldots, P(n)\right\}$. Note that the nonzero values among these predecessors show up in an increasing order, because $P^{+}$is monotonically increasing. Repeating the argument, it follows that the same permutation of the nonzero rows of $Y_{r}$ which makes that the nonzero entries in its last column appear in an increasing order, achieves that such a property holds for each of the columns of $Y_{r}$. Consequently, when all the columns of the row-permuted array $Y_{r}$ are stacked into a vector with $n m$ entries using the well-known vec $(\cdot)$ operator, a column vector remains which is equal to $(1,2, \ldots, n)^{T}$ when all the zeros entries are deleted.

Conversely, starting from the given vector of dynamical indices $d$ and an arbitrary choice of $\Pi$ permuting the nonzero rows of $Z$, the $n m$-vector with the given property and the right-aligned arrays $\Pi Y_{r}$ and $Y_{r}$ and the left-aligned array $Y$ are completely determined. The left-alignment property (i) of an admissible numbered Young diagram $Y$ is built-in. Properties (ii) and (iii) of $Y$ are not difficult to verify either, because they are easy for $\Pi Y_{r}$ and $Y_{r}$ and shifting the rows to move between $Y_{r}$ and $Y$ does not basically change the requirements (one only needs to take into account that zeros may occur to the left of a string of nonzero entries in $Y_{r}$, but the dynamical indices now specify the length of such a string in advance).

Note that the technique used in the proof of this proposition is constructive and can be used to generate all the admissible numbered Young diagrams corresponding to a given nice selection. There are $p_{B}$ ! different possibilities, where $p_{B}$ can be read off from $d$ as the number of dynamical indices $d_{i}>0$.

For given $m$ and $n$ and for each nice selection with a vector of dynamical indices $d$, one can consider the family $\mathcal{F}(d)$ of controllable pairs which have the additional property that the selected columns from the controllability matrix are linearly independent. Then we know that each controllable pair $(A, B)$ lies in at least one of the families $\mathcal{F}(d), d \in \mathcal{D}(m, n)$.

Now consider the family of all controllable pairs $(A, B)$ with A discrete-time asymptotically stable, and the question of how to parameterize this family up to state isomorphism. (I.e., up to multiplication of the controllability matrix $K$ by a nonsingular $n \times n$ matrix on the left.) Every such pair $(A, B)$ corresponds to a positive definite controllability Gramian $W_{c}$, which can be factored into $W_{c}=M^{T} M$ 
by making a well-defined choice for $M$, e.g. by prescribing it to be a positive upper triangular Cholesky factor. Such a choice can be parameterized to involve precisely $n(n+1) / 2$ independent real parameters. Using $M$ to act as a state isomorphism transforms $(A, B)$ into an input-normal controllable pair, but it does not affect any linear dependence relations between the columns of the controllability matrix $K$. Hence it allows one to restrict attention to the question of parameterizing the family of input-normal controllable pairs $(A, B)$ up to orthogonal state isomorphism.

Note that an input-normal controllable pair $(A, B)$ corresponds to a row-orthonormal partitioned matrix $[B, A]$ for which $A$ is asymptotically stable, and vice versa (see e.g. [7], [8]). Then for each admissible numbered Young diagram $Y$ the family of row-orthonormal $[B, A]$ with $A$ asymptotically stable and with an admissible pivot structure corresponding to $Y$, forms a local canonical form for this family. This set of local canonical forms is covering this family in the sense that for each roworthonormal $[B, A]$ with $A$ asymptotically stable there exists an admissible numbered Young diagram $Y$ and an orthogonal matrix $Q$ such that $\left[Q B, Q A Q^{T}\right]$ has the admissible pivot structure associated with $Y$. Furthermore, because of uniqueness of the associated QR-decomposition due to positive upper triangularity, for such a combination of $[B, A]$ and $Y$ the matrix $Q$ and hence $\left[Q B, Q A Q^{T}\right]$ is unique.

An interesting question is how to obtain a minimal sub-atlas of this atlas of local canonical forms, minimal in the sense that no further local canonical forms can be left out without losing the property of covering the family. To obtain a minimal sub-atlas we have to choose one of the local canonical forms for each $d \in \mathcal{D}(m, n)$. This implies that for each $d \in \mathcal{D}(m, n)$ we have to choose one of the $p_{B}$ ! possible numberings of the associated Young diagram. As each such numbering is associated with a permutation of the nonzero rows of the Young diagram this choice can be fixed by specifying that permutation. One possible choice is the unique permutation for which the permuted dynamical indices form a non-increasing sequence, while the order of the rows which have the same dynamical index is kept the same. Note that this permutation is used only to determine the numbering in the Young diagram, the ordering of the dynamical indices is left unchanged. With hindsight one can say that this particular choice to obtain a minimal atlas was used in [6] in a similar approach for continuous-time input-normal pairs and lossless systems. Just as in that paper for the continuous-time case, here each local canonical form on discrete-time asymptotically stable input normal systems defines a balanced local canonical form on minimal discrete-time lossless systems of order $n$. How these balanced local canonical forms for minimal discrete-time lossless systems of order $n$ are related to those constructed in [7] by means of the tangential Schur algorithm is the topic of the next section.

\section{Atlases of balanced canonical forms for lossless systems}

We now have two approaches to arrive at an atlas of overlapping balanced canonical forms for discretetime lossless systems: one using the balanced realizations associated with the tangential Schur algorithm and one based on balanced realizations with an imposed pivot structure on the row-orthonormal matrix $[B, A]$, hence on the orthogonal realization matrix $R$. However, one of our main results is that the second approach corresponds to making special choices for the direction vectors in the first approach. Hence the atlas of overlapping balanced canonical forms resulting from the second approach is a sub-atlas of the atlas of overlapping balanced canonical forms in the first approach. The precise formulation is as follows.

Theorem 5.1 Let $Y$ be an admissible numbered Young diagram, corresponding to an associated nice pivot structure $\widetilde{J}$ (for controllability matrices) and an admissible pivot structure $J$ (for $n \times(m+n)$ matrices). For each $k=1,2, \ldots, n$, choose the direction vector $u_{n+1-k}$ equal to $e_{i(k)}$, the $i(k)$-th 
standard basis vector in $\mathbb{R}^{m}$, where $(i(k), j(k))$ denotes the unique pair of indices such that $y_{i(k), j(k)}=$ $k$. Then for any choice of the Schur parameter vectors $v_{1}, v_{2}, \ldots, v_{n}$ (all of length $<1$ ) and for any choice of the orthogonal matrix $D_{0}$, consider the $(m+n) \times(m+n)$ orthogonal realization matrix $R$ given by (ब). It follows that $J$ is an admissible pivot structure for the sub-matrix $[B, A]$ and $\widetilde{J}$ is a nice pivot structure for the controllability matrix $K$.

A detailed technical proof of this theorem is given in Appendix B.

From the point of view of the tangential Schur algorithm, it is of interest also to directly characterize all the sequences of direction vectors $u_{1}, u_{2}, \ldots, u_{n}$ that give rise to an admissible pivot structure for the matrix $[B, A]$ (and an accompanying nice pivot structure for the controllability matrix $K$ ).

Theorem 5.2 Consider a chart associated with the tangential Schur algorithm (with all the interpolation points $w_{k}$ located at the origin), specified by a sequence of direction vectors $\left\{u_{1}, \ldots, u_{n}\right\}$. Then each $[B, A]$ resulting from this chart exhibits an admissible pivot structure, irrespective of the choice of Schur vectors $v_{1}, \ldots, v_{n}$, if the sequence of direction vectors consists of standard basis vectors, say $u_{k}=e_{\mu(k)}$ for some indices $\mu(1), \ldots, \mu(n)$ chosen from $\{1,2, \ldots, m\}$, satisfying the following condition:

for each $k=1,2, \ldots, n-1$, if there exists a largest index $\ell$ strictly less than $k$ such that $u_{\ell}=e_{\mu(k)}$, then $\mu(k+1)$ is from the set $\{\mu(\ell+1), \ldots, \mu(k)\}$.

Proof. This follows directly from the properties of the three procedures introduced in Appendix B to generate an admissible numbered Young diagram. Details are left to the reader.

Example. Consider the same situation as for the example in Appendix B, where $m=5, n=12$ and $\left(q_{1}, q_{2}, q_{3}, q_{4}, q_{5}\right)=(4,1,9,0,7)$. There it is remarked that the choice of direction vectors $u_{n+1-k}=$ $e_{i(k)}$ can be rewritten as $u_{k}=e_{\mu(k)}$ where $\mu(k)$ denotes the index of the value 1 in the vector $\eta_{k}$, generated by the 'third procedure'. In this example it follows that the sequence $\left\{u_{1}, u_{2}, \ldots, u_{12}\right\}$ is given by $\left\{e_{2}, e_{5}, e_{1}, e_{3}, e_{2}, e_{5}, e_{1}, e_{2}, e_{1}, e_{2}, e_{2}, e_{2}\right\}$. Note that this sequence satisfies the condition of Theorem 5.2 for all $k=1,2, \ldots, 11$. E.g., for $k=6$ the previous occurrence of the vector $u_{6}=e_{5}$ happened for $\ell=2$. The condition of the theorem requires $u_{7}$ to occur in the set $\left\{u_{3}, u_{4}, u_{5}, u_{6}\right\}=$ $\left\{e_{1}, e_{3}, e_{2}, e_{5}\right\}$, which indeed is the case.

\section{Conclusions and discussion}

In this paper we have developed a detailed procedure to construct an atlas of overlapping (local) balanced canonical forms for MIMO discrete-time lossless systems $(A, B, C, D)$ of a given order $n$. To achieve this, the concept of an admissible pivot structure for $[B, A]$ has been introduced, which induces a full (nice) pivot structure for the associated controllability matrix $K$. The approach taken in Sections 3 and 4 has a wider range of applicability though: it builds on the algebraic relationship between $[B, A]$ and $K$, and it neither relies on input-normality of $(A, B)$ nor on the discrete-time setting for the lossless systems. When one is dealing with a system having a special (non-generic) Kronecker structure, this can be recognized in these local canonical forms by certain entries becoming zero. To demonstrate the structure of the charts that constitute the atlases discussed in this paper, a detailed worked example is included in Appendix C.

One of the main practical reasons for studying overlapping canonical forms is that (iterative) identification and optimization algorithms in the MIMO case may suffer from numerical ill-conditioning 
and slow convergence when they pass by systems that not too far from systems with a non-generic structure. Switching charts may then help to improve the algorithmic performance. The connection in Section 5 with the atlas of charts developed for discrete-time input-normal pairs involving the tangential Schur algorithm is useful, because that set-up involves (well conditioned!) orthogonal matrix computations while it is tailored to deal with the important class of stable systems. The tangential Schur algorithm provides one with a lot of flexibility to design local canonical forms. Since it is computationally expensive to switch charts at each and every iteration, a suitably chosen finite sub-atlas is welcome. In the present paper we have indicated the restrictions that should be taken into account when choosing direction vectors $u_{k}$ from the set of standard basis vectors, if a pivot structure is to show up not only in $[B, A]$ but also in the controllability matrix $K$. When a nice pivot structure is present in $K$, this has the advantage that the impact of state vector truncation is easier to analyze; controllability is then preserved. This is of importance in the context of model order reduction applications.

Future research addresses the issue of monitoring the conditioning of a chart (i.e., a local canonical form) at a given system, and the issue of selecting a better chart when switching becomes necessary. Since the total number of charts in an atlas quickly grows large with the dimensions $m$ and $n$ (even for the case of admissible pivot structures) it may not be attractive to carry out a full search for a better chart over the entire atlas. The rank structure in $K$ can then be instrumental in designing a quick online algorithm which guarantees a certain degree of conditioning improvement. This is currently under investigation.

\section{References}

[1] J. Bruls, C.T. Chou, B.R.J. Haverkamp, and M. Verhaegen. Linear and non-linear system identification using separable least-squares. European Journal of Control, 5:116-128, 1999.

[2] W. Fulton. Young Tableaux with Applications to Representation Theory and Geometry. Cambridge University Press, 1997.

[3] Y. Genin, P. Van Dooren, T. Kailath, J.-M. Delosme, and M. Morf. On $\Sigma$-lossless transfer functions and related questions. Linear Algebra and its Applications, 50:251-275, 1983.

[4] B. Hanzon. Identifiability, recursive identification and spaces of linear dynamical systems, 1989. CWI Tracts 63,64, CWI Amsterdam.

[5] B. Hanzon. A new balanced canonical form for stable multivariable systems. IEEE Transactions on Automatic Control, AC-40:374-378, 1995.

[6] B. Hanzon and R.J. Ober. Overlapping block-balanced canonical forms for various classes of linear systems. Linear Algebra and its Applications, 281:171-225, 1998.

[7] B. Hanzon, M. Olivi, and R.L.M. Peeters. Balanced realizations of discrete-time stable all-pass systems and the tangential Schur algorithm. Linear Algebra and its Applications, 418:793-820, 2006.

[8] B. Hanzon and R.L.M. Peeters. Balanced parametrizations of stable SISO all-pass systems in discrete-time. Mathematics of Control, Signals, and Systems, 13:240-276, 2000.

[9] T. Kailath. Linear Systems. Prentice-Hall, 1980. 
[10] B.C. Moore. Principal component analysis in linear systems: Controllability, observability, and model reduction. IEEE Transactions on Automatic Control, AC-26:17-32, 1981.

[11] R.J. Ober. Balanced realizations: canonical form, parametrization, model reduction. International Journal of Control, 46:643-670, 1987.

[12] R.J. Ober. Balanced canonical forms. In S. Bittanti, editor, Identification, Adaptation and Learning. Springer Verlag, 1996.

\section{A Proof of part (b) of Theorem 3.4}

Consider a non-admissible full pivot structure $J=\left\{j_{1}, j_{2}, \ldots, j_{n}\right\}$ for $[B, A]$. Then it either holds that: (1) $J$ does not prescribe $B$ to have have a pivot-1 column, or (2) $J$ does prescribe $B$ to have a pivot-1 column, but $J$ does not impose a staircase structure on $A$.

In case (1) it holds that $j_{1}>m$. We distinguish between two situations. (i) If $j_{1}=m+1$, then the first column of $A$ is a pivot- 1 column. Then consider the following example: for each $k=1, \ldots, n$ let column $j_{k}$ of $[B, A]$ be equal to the $k$-th standard basis vector $e_{k} \in \mathbb{R}^{n}$ and let the remaining $m$ columns of $[B, A]$ all be zero. Clearly, $[B, A]$ exhibits the given full pivot structure $J$, but $e_{1}^{T} B=0$ and $e_{1}^{T} A=e_{1}^{T}$ so that $e_{1}^{T} K=0$. Hence $K$ does not have a pivot- 1 column, so it does not have a full pivot structure.

(ii) If instead $j_{1}>m+1$, then consider the following example: for each $k=1, \ldots, n$ choose column $j_{k}$ of $[B, A]$ to be a positive pivot- $k$ vector and let the remaining $m$ columns of $[B, A]$ all be chosen to be strictly positive (so that effectively they are all positive pivot- $n$ vectors). Clearly, $[B, A]$ exhibits the given full pivot structure $J$. Note that each column in $B$ is (effectively) a positive pivot- $k$ vector with $k \geq 2$. Now, if $v$ is a positive pivot- $k$ vector, then $A v$ is a positively weighted linear combination of the first $k$ columns of $A$. Since all columns of $A$ are (effectively) positive pivot- $\ell$ vectors for certain values of $\ell$, the vector $A v$ is a positive pivot- $p$ vector where $p$ is the maximal (effective) pivot position among the first $k$ columns of $A$. Now, the first column of $A$ has at least two nonzero entries, because $j_{1}>m+1$. Therefore, each column of $A B$ is (effectively) a positive pivot- $p$ vector with $p \geq 2$. By induction it follows that all columns of $K$ are (effectively) positive pivot- $p$ vectors with $p \geq 2$. Consequently, $K$ does not have a pivot- 1 column, so it does not have a full pivot structure.

In case (2) it holds that $j_{1} \leq m$, but the staircase structure does not necessarily hold for $A$. We again distinguish between two situations. (i) Suppose that for some $k<n$ there is a pivot- $k$ vector in $A$ for which there is either a non-pivot column in $A$ preceding it, or a pivot- $\ell$ vector preceding it with $\ell>k$. Then consider basically the same example as used in case (1) part (ii): for each $k=1, \ldots, n$ choose column $j_{k}$ of $[B, A]$ to be a positive pivot- $k$ vector and let the remaining $m$ columns of $[B, A]$ all be chosen to be strictly positive (so that effectively they are all positive pivot- $n$ vectors). For this example it now follows that $K$ does not have a pivot- $k$ vector, because $B$ does not have one and because for all $p=1, \ldots, n$ the maximum (effective) pivot position among the first $p$ columns of $A$ can never be equal to $k$.

(ii) For all $k<n$ the pivot- $k$ vectors in $A$ respect the staircase structure, but there is a prescribed pivot- $n$ vector in $A$ which is directly preceded by a non-pivot column. If this pivot- $n$ vector occurs in the last column of $A$, then one may consider the same kind of example as used in case (1) part (i): for each $k=1, \ldots, n$ let column $j_{k}$ of $[B, A]$ be equal to $e_{k}$ and let the remaining $m$ columns of $[B, A]$ all be zero. Now $e_{n}^{T} B=0$ and $e_{n}^{T} A=e_{n}^{T}$ so that $e_{n}^{T} K=0$. Hence $K$ does not have a pivot- $n$ column, so it does not have a full pivot structure. If the pivot- $n$ vector does not occur in the last column of $A$, 
then the last column of $A$ is a non-pivot column. Summarizing, we then are in a situation where the first $p_{A}-1$ columns of $A$ exhibit a staircase structure, column $p:=j_{n}-m>p_{A}$ of $A$ is a pivot- $n$ column and the two columns $p-1$ and $n$ of $A$ are both non-pivot columns. Then consider the following example. Column $j_{p-1}$ of $[B, A]$ is defined as the pivot- $(p-1)$ vector $\frac{3}{5} e_{p-1}$. Column $j_{p}$ of $[B, A]$ is defined as the pivot- $p$ vector $\frac{3}{5} e_{p}+\frac{16}{25} e_{p-1}$. The $3 \times 3$ sub-matrix $S$ of $A$ constituted by the intersection of its rows and columns with indices $p-1, p$ and $n$ is defined as $S=\left(\begin{array}{ccc}0 & \frac{36}{125} & -\frac{48}{125} \\ 0 & -\frac{12}{25} & \frac{16}{25} \\ -\frac{3}{5} & \frac{16}{25} & \frac{12}{25}\end{array}\right)$. All remaining entries at pivot positions in $[B, A]$ are defined to be 1 and all other entries are set to zero. For this example it will be shown that the entries in the last row of $K$ are never positive, so that $K$ does not have a full pivot structure. Note that all columns in $B$ have a last entry that is equal to zero. All other columns in $K$ are of the form $A v$ for some vector $v \in \mathbb{R}^{n}$. For $A v$ to have a nonzero last entry, at least one of the entries in positions $p-1, p$ and $n$ of $v$ must be nonzero. Such vectors $v$ must come from repeated pre-multiplication of the columns of $B$ by the matrix $A$. The first vectors to have such a structure are the pivot- $(p-1)$ vector and the pivot- $p$ vector that both occur among the columns of $B$ and the first $p_{A}-1$ columns of $A$. Once such vectors $v$ are multiplied by $A$, only the entries in positions $p-1, p$ and $n$ can become nonzero: the subspace spanned by $e_{p-1}, e_{p}$ and $e_{n}$ is an invariant subspace of $A$. Restricting to this subspace, the matrix $A$ is represented by the sub-matrix $S$ given above. Consequently, the entries in the last row of $K$ are either zero or obtained as the entries in the last row of the controllability matrix of the pair $(T, S)$ with $T=\left(\begin{array}{cc}\frac{3}{5} & \frac{16}{25} \\ 0 & \frac{3}{5} \\ 0 & 0\end{array}\right)$ which represents the pivot- $(p-1)$ and the pivot- $p$ vector in this new notation. For the matrix $S$ it is easily established that $S^{3}=\frac{544}{625} S$. It therefore suffices to compute the last row of the matrix $\left[T, S T, S^{2} T\right]$, which is equal to $\left(0,0,-\frac{9}{25}, 0,-\frac{108}{625},-\frac{36}{125}\right)$. This proves that all these entries are indeed non-positive.

\section{Remark.}

In the case of balanced realizations of lossless systems we will in addition require $[B, A]$ to have orthonormal rows. The proof above does not entirely apply to this restricted situation. For example if $[B, A]$ only has non-negative entries then orthogonality of the rows requires that in each column there is at most one nonzero entry. This requirement is violated by the counterexamples presented in case (1) part (ii) and in case (2) part (i) of the proof, because non-pivot columns are chosen to be positive pivot- $n$ vectors. How to obtain a proof for this more restricted orthonormal case is an open problem at this point. Note however that the counterexamples presented in case (1) part (i) and in case (2) part (ii) of the proof have in fact already been designed to involve $[B, A]$ with orthonormal rows.

\section{B Proof of Theorem 5.1]}

To prove this theorem it is helpful first to reconsider the precise relationship between $Y, \widetilde{J}, J$ and the pivot structure $\left\{q_{1}, \ldots, q_{m}\right\}$ of the matrix $B$. Recall that the number 1 appears in the pivot structure $\left\{q_{1}, \ldots, q_{m}\right\}$ for $B$ because of admissibility and this sequence completely characterizes the successor function $S$, the column-oriented description $Q$, the admissible pivot structure $J$, the nice pivot structure $\widetilde{J}$ and the admissible numbered Young diagram $Y$ in the way explained before.

We have previously introduced the following construction procedure for $Y$ from $\left\{q_{1}, \ldots, q_{m}\right\}$ : 


\section{Procedure 1}

(a) Construction: the successor function $S$ is defined as the increasing sequence of all positive numbers in $\{1,2, \ldots, n\}$ not occurring in $\left\{q_{1}, \ldots, q_{m}\right\}$ completed by a sequence of $p_{B}$ zeros; in addition $S(0)=0$.

(b) Initialization: set $y_{i, 1}:=q_{i}$ for $i=1, \ldots, m$.

(c) Recursion: set $y_{i, j+1}:=S\left(y_{i, j}\right)$ for $i=1, \ldots, m$ and $j=1, \ldots, n-1$.

A second way to generate $Y$ in a dynamical fashion which avoids the explicit construction of $S$, is by means of the following procedure:

\section{Procedure 2}

(a) Initialization: set $y_{i, 1}=q_{i}$ for $i=1, \ldots, m$.

(b) Recursion: for $k=1,2, \ldots, n$, if the value $k$ has not yet been assigned to an entry of $Y$ then select the smallest nonzero number $y_{i, j}$ in $Y$ for which the entry $y_{i, j+1}$ immediately to its right has still not been assigned some value and set $y_{i, j+1}=k$.

(c) Termination: set all the remaining entries of $Y$ equal to zero.

It is not very hard to establish that the array $Y$ constructed in this fashion is indeed admissible and identical to the array $Y$ constructed previously with the help of $S$.

A third way to generate $Y$ from $\left\{q_{1}, \ldots, q_{m}\right\}$ employs a sequence of vectors $\eta_{k}(k=0,1,2, \ldots, n)$ and proceeds as follows in a backward fashion:

\section{Procedure 3}

(a) Initialization: set $\eta_{n}=\left(q_{1}, \ldots, q_{m}\right)^{T}$.

(b) Backward recursion: for $k=n-1, \ldots, 1,0$ construct $\eta_{k}$ from $\eta_{k+1}$ by executing the following three rules in the given order:

(1) if $\left(\eta_{k+1}\right)_{i}=0$ then set $\left(\eta_{k}\right)_{i}:=0$;

(2) if $\left(\eta_{k+1}\right)_{i}>1$ then set $\left(\eta_{k}\right)_{i}:=\left(\eta_{k+1}\right)_{i}-1$;

(3) if $\left(\eta_{k+1}\right)_{i}=1$ then define $\xi_{k}$ as the smallest positive number different from all the entries of $\eta_{k}$ already assigned by rules (1) and (2); if $\xi_{k} \leq k$ then set $\left(\eta_{k}\right)_{i}:=\xi_{k}$ else set $\left(\eta_{k}\right)_{i}:=0$.

(c) Construction: for each $i=1, \ldots, m$ consider the $d_{i}$ values of $k$ for which $\left(\eta_{n+1-k}\right)_{i}=1$ and assign these values (in increasing order) to the first $d_{i}$ entries of row $i$ of $Y$; set all other entries to 0 .

The validity of this third procedure for generating $Y$ can be seen as follows. First, note that because of rule (2) in each recursion step (b), the first column of $Y$ attains the required form containing $q_{1}, \ldots, q_{m}$, since the number 1 first occurs in position $i$ of $\eta_{n+1-k}$ for $k=q_{i}$. Also note that the positive integers in $\eta_{n}$ are all different (since this holds for $q_{1}, \ldots, q_{m}$ ) and that the rules in each recursion step (b) are such that this property is preserved for all vectors $\eta_{k}$. Next, these rules are such that each vector $\eta_{k}$ has precisely one entry equal to 1 , for all $k=1, \ldots, n$. The construction in step (c) is such that all the numbers from $\{1, \ldots, n\}$ show up precisely once in a corresponding left-aligned numbered Young diagram $Y$. Finally, rule (3) in each recursion step (b) guarantees that $y_{i, j}$ is followed by $y_{i, j+1}=S\left(y_{i, j}\right)$ : note that $y_{i, j}=k$ is equivalent to $\left(\eta_{n+1-k}\right)_{i}=1$ and $\left(\eta_{n-k}\right)_{i}=\xi_{k}$ implies that $y_{i, j+1}=k+\xi_{k}$; here $\xi_{k}>0$ is chosen as small as possible, precisely in line with the second procedure for generating $Y$.

Example. Consider the situation with $m=5, n=12$ and $\left(q_{1}, q_{2}, q_{3}, q_{4}, q_{5}\right)=(4,1,9,0,7)$. Then the successor function $S$ is described by $\left\{s_{1}, s_{2}, \ldots, s_{10}\right\}=\{2,3,5,6,8,10,11,12,0,0,0,0\}$. It 
follows that $p_{B}=4$ and the corresponding admissible numbered Young diagram $Y$ is given by:

$Y=$\begin{tabular}{|l|l|l|l|l|l|l|l|l|l|l|l|}
\hline 4 & 6 & 10 & & & & & & & & & \\
\hline 1 & 2 & 3 & 5 & 8 & 12 & & & & & & \\
\hline 9 & & & & & & & & & & & \\
\hline & & & & & & & & & & & \\
\hline 7 & 11 & & & & & & & & & & \\
\hline
\end{tabular}

where all the zeros are omitted for clarity. The second procedure for generating $Y$ without the explicit construction of the successor function $S$ yields the same result. It proceeds from the given first column of $Y$ by putting the value 2 after the value 1 , then the value 3 after the value 2 , then the value 5 after the value 3 , then the value 6 after the value 4 , and so on. The values 1, 4, 7 and 9 are skipped, because they have already been assigned to the first column of $Y$.

The third procedure for generating $Y$ involves the backward recursion for the construction of the vectors $\eta_{k}$, for $k=n, n-1, \ldots, 1,0$. This produces the following sequence:

\begin{tabular}{|c|c|c|c|c|c|c|c|c|c|c|c|c|}
\hline$\eta_{12}$ & $\eta_{11}$ & $\eta_{10}$ & $\eta_{9}$ & $\eta_{8}$ & $\eta_{7}$ & $\eta_{6}$ & $\eta_{5}$ & $\eta_{4}$ & $\eta_{3}$ & $\eta_{2}$ & $\eta_{1}$ & $\eta_{0}$ \\
\hline 4 & 3 & 2 & 1 & 2 & 1 & 4 & 3 & 2 & 1 & 0 & 0 & 0 \\
1 & 1 & 1 & 2 & 1 & 3 & 2 & 1 & 4 & 3 & 2 & 1 & 0 \\
9 & 8 & 7 & 6 & 5 & 4 & 3 & 2 & 1 & 0 & 0 & 0 & 0 \\
0 & 0 & 0 & 0 & 0 & 0 & 0 & 0 & 0 & 0 & 0 & 0 & 0 \\
7 & 6 & 5 & 4 & 3 & 2 & 1 & 4 & 3 & 2 & 1 & 0 & 0 \\
\hline
\end{tabular}

For instance, the vector $\eta_{7}$ is obtained from the vector $\eta_{8}$ as follows. First all the entries equal to zero are copied and all the values $\left(\eta_{8}\right)_{i} \geq 2$ are decreased by 1 to produce the corresponding values of $\left(\eta_{7}\right)_{i}$. The value of $\left(\eta_{7}\right)_{2}$ is addressed last, because $\left(\eta_{8}\right)_{2}=1$. At that stage the values 1 and 2 have already been assigned to some entries of $\eta_{7}$ and it holds that $\xi_{7}=3$. Because $\xi_{7}=3$ is not larger than the index $k=7$, this value is assigned to $\left(\eta_{7}\right)_{2}$.

Once the vectors $\eta_{12}, \eta_{11}, \ldots, \eta_{1}$ have been constructed, the array $Y$ is constructed by considering the positions of the entries 1 . For the first row, these positions are subsequently 4,6 and 10 (proceeding in the given order from $\eta_{12}$ to $\eta_{1}$ corresponding to the index $n+1-k$ ). For the second row we have: $1,2,3,5,8$ and 12 , and so on.

The third way of characterizing $Y$ in terms of the pivot structure $\left\{q_{1}, \ldots, q_{m}\right\}$ has a number of properties that are worth noting in view of the proof of Theorem 5.1 below. First, note that rule (3) in step (b) implies that the maximum value among the entries of $\eta_{k}$ is at most $k$. (Therefore, $\eta_{0}$ is the zero vector.) Second, all the positive entries of a vector $\eta_{k}$ are different. This makes that if $\left(\eta_{k+1}\right)_{i}=1$ then a positive value $\xi_{k}$ is assigned to $\left(\eta_{k}\right)_{i}$ for $k \geq p_{B}$ and the value 0 is assigned for $k<p_{B}$. Third, note that the sequence of values $\xi_{n-1}, \xi_{n-2}, \ldots, \xi_{p_{B}}$ (in that backward order) is increasing. Fourth, the choice of direction vectors $u_{n+1-k}=e_{i(k)}$ can be rewritten as $u_{k}=e_{\mu(k)}$ where $\mu(k)$ denotes the index of the value 1 in the vector $\eta_{k}$. Note that according to this notation, $\left(\eta_{k}\right)_{\mu(k+1)}=\xi_{k}$ for $k=n-1, n-2, \ldots, p_{B}$. Finally, it will be shown that the vectors $\eta_{k}$ represent the pivot structures for the sequence of lossless systems of orders $k=1,2, \ldots, n$ encountered in the tangential Schur algorithm for the particular choice of direction vectors specified in Theorem 5.1.

Proof of Theorem 5.1. Consider the matrix product

$$
R=\Gamma_{n} \cdots \Gamma_{1} R_{0} \Delta_{1}^{T} \cdots \Delta_{n}^{T} .
$$


Note that the product $\Gamma_{n} \cdots \Gamma_{1} R_{0}$ is positive $m$-upper Hessenberg for any choice of Schur vectors $v_{1}, \ldots, v_{n}$. Post-multiplication by the matrix $\Delta_{1}^{T}$ only affects the last $m+1$ columns, because the matrix $\Delta_{1}^{T}$ is given by

$$
\Delta_{1}^{T}=\left[\begin{array}{ccc}
I_{n-1} & 0 & 0 \\
0 & e_{\mu(1)}^{T} & 0 \\
0 & I_{m}-e_{\mu(1)} e_{\mu(1)}^{T} & e_{\mu(1)}
\end{array}\right]
$$

where $\mu(1)$ denotes the location of the entry 1 in the vector $\eta_{1}$ which features in the third method for the construction of $Y$ from $\left\{q_{1}, \ldots, q_{m}\right\}$. The precise effect is as follows:

(i) column $n$ of $\Gamma_{n} \cdots \Gamma_{1} R_{0}$ (having a pivot in its last position) is moved into column $n+\mu(1)-1$;

(ii) column $n+\mu(1)$ of $\Gamma_{n} \cdots \Gamma_{1} R_{0}$ is moved into column $n+m$;

(iii) columns $n+1, \ldots, n+\mu(1)-1$ and $n+\mu(1)+1, \ldots, n+m$ of $\Gamma_{n} \cdots \Gamma_{1} R_{0}$ are moved one position to the left, into columns $n, \ldots, n+\mu(1)-2$ and $n+\mu(1), \ldots, n+m-1$, respectively.

Note that the last row of $\Gamma_{n} \cdots \Gamma_{1} R_{0} \Delta_{1}^{T}$ can be regarded to have the structure:

$$
\left[\begin{array}{lll}
0 & B_{1} & A_{1}
\end{array}\right]
$$

with $A_{1}$ of size $1 \times 1$ and $B_{1}$ of size $1 \times m$. The $1 \times(m+1)$ partitioned matrix [ $\left.B_{1}, A_{1}\right]$ has an admissible pivot structure for which the column-oriented pivot structure of $B_{1}$ is given by $\{0, \ldots, 0,1,0, \ldots, 0\}$ with the value 1 in position $\mu(1)$. In other words, the pivot structure of $B_{1}$ is described by $\eta_{1}$.

Consider the last $k$ rows of the matrix product $\Gamma_{n} \cdots \Gamma_{1} R_{0} \Delta_{1}^{T} \cdots \Delta_{k}^{T}$. Note that these can be regarded to constitute the structure:

$$
\left[\begin{array}{lll}
0 & B_{k} & A_{k}
\end{array}\right]
$$

with $A_{k}$ of size $k \times k$ and $B_{k}$ of size $k \times m$. Now suppose that the $k \times(m+k)$ partitioned matrix $\left[B_{k}, A_{k}\right]$ is known to have an admissible pivot structure for which the column-oriented pivot structure of $B_{k}$ is given by the vector $\eta_{k}$. (This is the induction hypothesis.) We consider what happens under post-multiplication by the matrix $\Delta_{k+1}^{T}$. Note that this matrix is given by:

$$
\Delta_{k+1}^{T}=\left[\begin{array}{cccc}
I_{n-k-1} & 0 & 0 & 0 \\
0 & e_{\mu(k+1)}^{T} & 0 & 0 \\
0 & I_{m}-e_{\mu(k+1)} e_{\mu(k+1)}^{T} & e_{\mu(k+1)} & 0 \\
0 & 0 & 0 & I_{k}
\end{array}\right]
$$

Therefore, post-multiplication by $\Delta_{k+1}^{T}$ only acts on the columns $n-k, \ldots, n-k+m$ of the matrix $\Gamma_{n} \cdots \Gamma_{1} R_{0} \Delta_{1}^{T} \cdots \Delta_{k}^{T}$.

The partitioned matrix $\left[B_{k+1}, A_{k+1}\right]$ is then formed as

$$
\left[B_{k+1}, A_{k+1}\right]=\left[\begin{array}{ccc}
\gamma & \beta & \alpha \\
0 & B_{k} & A_{k}
\end{array}\right]\left[\begin{array}{ccc}
e_{\mu(k+1)}^{T} & 0 & 0 \\
I_{m}-e_{\mu(k+1)} e_{\mu(k+1)}^{T} & e_{\mu(k+1)} & 0 \\
0 & 0 & I_{k}
\end{array}\right]
$$

where $\gamma$ is a positive scalar and $\beta$ and $\alpha$ are $1 \times m$ and $1 \times k$ row vectors, respectively. It follows that the post-multiplying matrix carries out the following action:

(i) the columns involving $A_{k}$ remain unchanged;

(ii) column 1 of $\left[\begin{array}{ccc}\gamma & \beta & \alpha \\ 0 & B_{k} & A_{k}\end{array}\right]$ having a pivot in its first position, is moved into column $\mu(k+1)$; 
(iii) column $\mu(k+1)+1$ of $\left[\begin{array}{ccc}\gamma & \beta & \alpha \\ 0 & B_{k} & A_{k}\end{array}\right]$ is moved into column $m+1$;

(iv) columns $2, \ldots, \mu(k+1)$ and $\mu(k+1)+2, \ldots, m+1$ of $\left[\begin{array}{ccc}\gamma & \beta & \alpha \\ 0 & B_{k} & A_{k}\end{array}\right]$ are moved one position to the left, into columns $1, \ldots, \mu(k+1)-1$ and $\mu(k+1)+1, \ldots, m$, respectively.

This shows that the pivot structure of $B_{k+1}$ is obtained from the pivot structure of $B_{k}$ in the following way: all the nonzero entries of the structure vector $\eta_{k}$ are increased by 1 except for the entry with index $\mu(k+1)$, which is reset to 1 . This means that the pivot structure of $B_{k+1}$ is indeed given by the vector $\eta_{k+1}$.

It remains to show that the matrix $A_{k+1}$ again has a staircase form. Now, $A_{k}$ has a staircase form according to the induction hypothesis and $A_{k+1}$ is recognized to be of the form

$$
A_{k+1}=\left[\begin{array}{cc}
\delta & \alpha \\
\epsilon & A_{k}
\end{array}\right]
$$

where $\delta$ is the $\mu(k+1)$-st entry of the row vector $\beta$ and $\epsilon$ is the $\mu(k+1)$-st column of $B_{k}$. This means that the pivot in the first column of $A_{k+1}$ shows up in position $\left(\eta_{k}\right)_{\mu(k+1)}+1$, which is equal to $\xi_{k}+1$. However, it has already been established that the sequence $\xi_{n-1}, \xi_{n-2}, \ldots, \xi_{p_{B}}$ is increasing. Therefore, $A_{k+1}$ also has a staircase form.

By induction this shows for all $k=1,2, \ldots, n$, that $\left[B_{k}, A_{k}\right]$ has an admissible pivot structure for which the vector $\eta_{k}$ specifies the pivot structure of the matrix $B_{k}$. In particular, for $k=n$ the claim of the theorem follows.

\section{An atlas for input-normal pairs $(A, B)$ under orthogonal state-space equivalence, with $m=3$ and $n=4$}

To illustrate the results and constructions of this paper, we here present an atlas for the manifold of (controllable) input-normal pairs $(A, B)$ under orthogonal state-space equivalence, for the non-trivial case $m=3$ and $n=4$. Each of the charts in this atlas gives rise to a particular full pivot structure in the controllability matrix $K$ and an admissible pivot structure for the row-orthonormal matrix $[B, A]$.

For given $m$ and $n$, the number of different admissible numbered Young diagrams (see the end of Section (3) is specified by $\sum_{\ell=1}^{\min \{m, n\}} \ell !\left(\begin{array}{c}m \\ \ell\end{array}\right)\left(\begin{array}{c}n-1 \\ \ell-1\end{array}\right)$. For the case $m=3$ and $n=4$ this amounts to 39 . To obtain a minimal sub-atlas, precisely one chart should be included for each nice selection, i.e. for each vector of dynamical indices $d$ in $\mathcal{D}(m, n)$. The cardinality of $\mathcal{D}(m, n)$ is easily computed as $\left(\begin{array}{c}m+n-1 \\ m-1\end{array}\right)$. For the case $m=3$ and $n=4$ this implies that a minimal sub-atlas consists of 15 charts. In Tables 13 the 15 different vectors of dynamical indices for this example are displayed, along with the corresponding 39 admissible numbered Young diagrams and their associated pivot structures in $K$ and in $[B, A]$.

To arrive at an explicit parameterization of a chart in these tables, one may proceed in the discretetime case by exploiting Eqn. (9) for the construction of orthogonal realization matrices, corresponding to balanced realizations of discrete-time lossless systems. Here the sequence of direction vectors $\left\{u_{1}, u_{2}, u_{3}, u_{4}\right\}$ is chosen to consist of particular standard basis vectors, as indicated for each chart in these tables too. The parameters are then provided by the sequence of Schur vectors $\left\{v_{1}, v_{2}, v_{3}, v_{4}\right\}$ which are all required to be of length $<1$. The $3 \times 3$ orthogonal matrix block $D_{0}$ can be set to 
any fixed value; the choice $D_{0}=I_{3}$ is a convenient one. The latter is a consequence of the general fact that if an orthogonal realization matrix $R=\left[\begin{array}{cc}D & C \\ B & A\end{array}\right]$ is generated by Eqn. (9) for some $D_{0}$, $\left\{u_{1}, \ldots, u_{n}\right\}$ and $\left\{v_{1}, \ldots, v_{n}\right\}$, then the alternative choices $I_{m},\left\{u_{1}, \ldots, u_{n}\right\}$ and $\left\{D_{0}^{T} v_{1}, \ldots, D_{0}^{T} v_{n}\right\}$ yield the realization matrix $\left[\begin{array}{cc}D_{0} D & D_{0} C \\ B & A\end{array}\right]$, which exhibits exactly the same input pair $(A, B)$.

From such a (minimal or non-minimal) atlas for input-normal pairs under orthogonal state-space equivalence, a corresponding atlas for all input-normal pairs of the given dimensions $m$ and $n$ is directly obtained by regarding the associated manifold as a Cartesian product of the previous manifold with the orthogonal group $\mathcal{O}(n)$, related to the choice of state-space transformation.

To arrive at a corresponding atlas for $m \times m$ lossless systems of order $n$ one may instead regard this space as a Cartesian product of the previous manifold with the orthogonal group $\mathcal{O}(m)$, now related to the choice of $D_{0}$.

To obtain an atlas for asymptotically stable discrete-time systems of order $n$ with $m$ inputs and $p$ outputs, one may proceed by taking all the entries of $C$ and $D$ (of sizes $p \times n$ and $p \times m$, respectively) to be free parameters, only subject to the constraint that observability needs to hold for the pair $(C, A)$ (a property which is then generically satisfied in each chart, i.e. it only excludes a thin subset of parameter vectors). Such an approach is useful in system identification, for instance in conjunction with the method of separable least-squares (see [1]). Then we may have to consider output-normal forms instead, but this can be achieved easily using input-output duality.

Finally, to deal with the continuous-time case, the well-known bilinear transform can of course be applied. However, this will in general destroy the pivot structure in $K$ and in $[B, A]$. To employ the results directly in the continuous-time case too, note that the pivot structures for (controllable) inputnormal pairs $[B, A]$ as given in the Tables $1-3$ do in fact apply to the continuous-time case already, giving rise to local canonical forms that can be computed numerically for a given state-space realization in a straightforward way. What at present seems to be lacking in the continuous-time case is an explicit parameterization of these local canonical forms (such as may be required in system identification). This is currently the topic of ongoing research. 


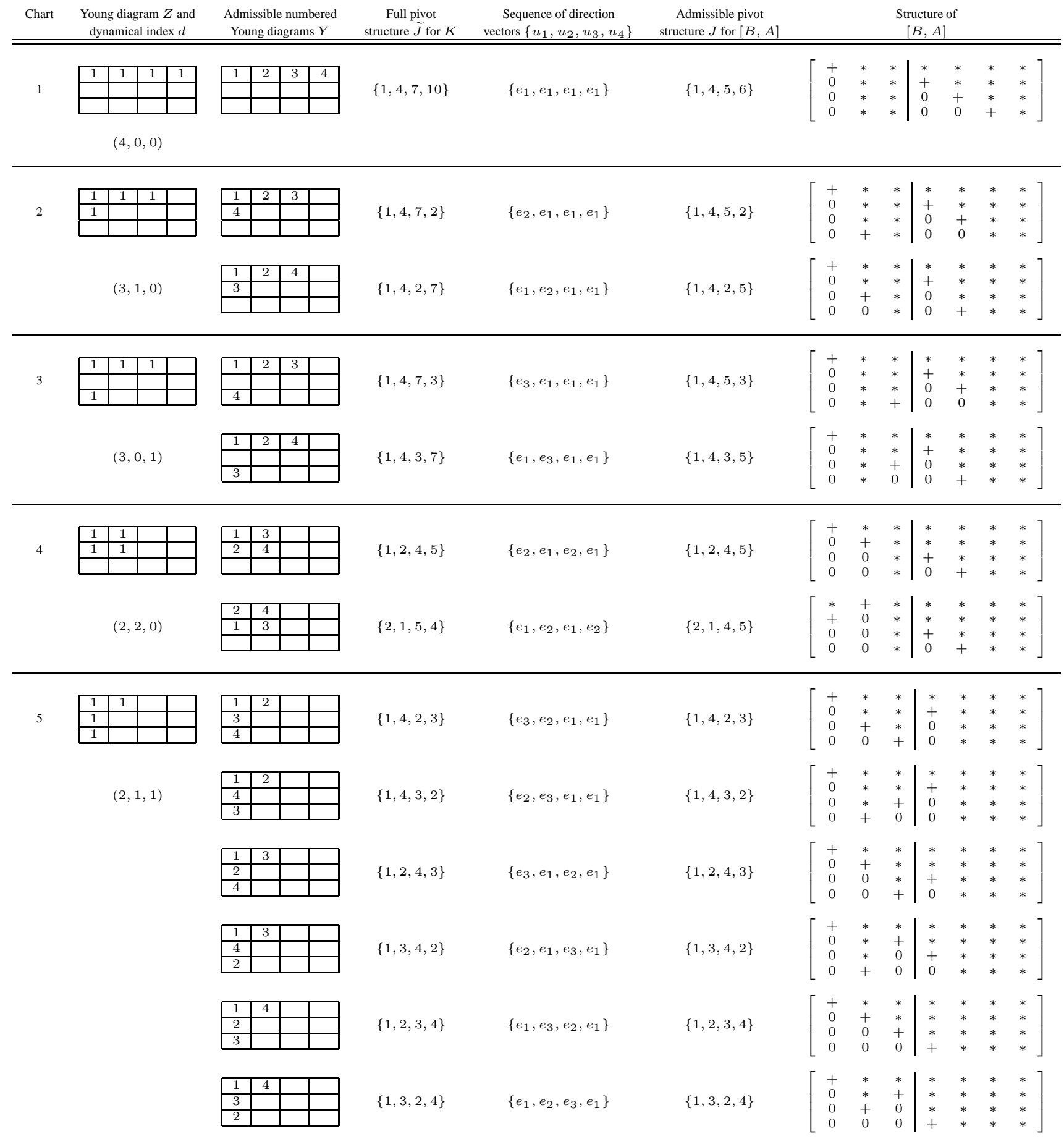

Table 1: Charts $1-5$ for $[B, A]$, for the case $m=3$ and $n=4$. 


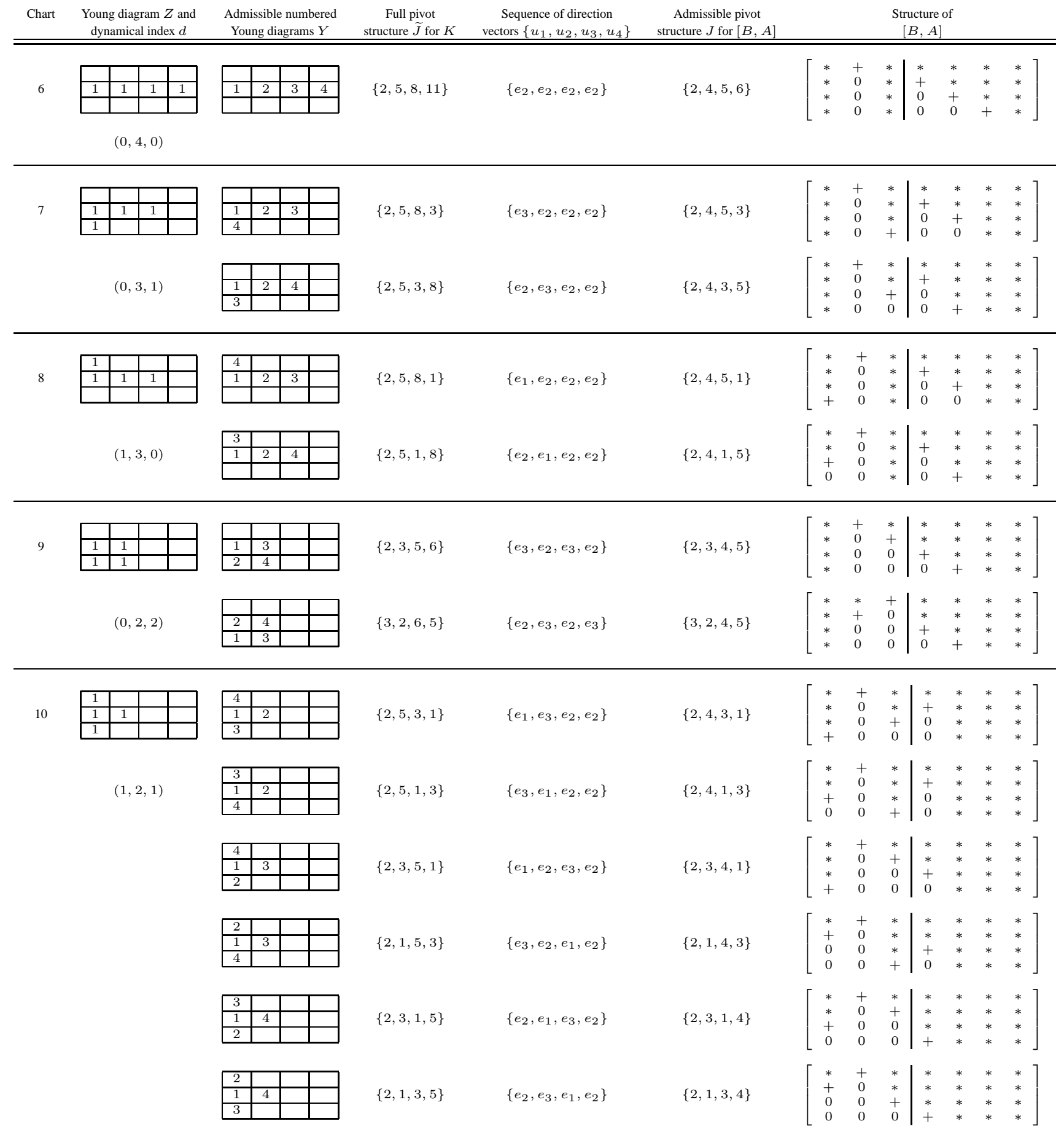

Table 2: Charts 6-10 for $[B, A]$, for the case $m=3$ and $n=4$. 


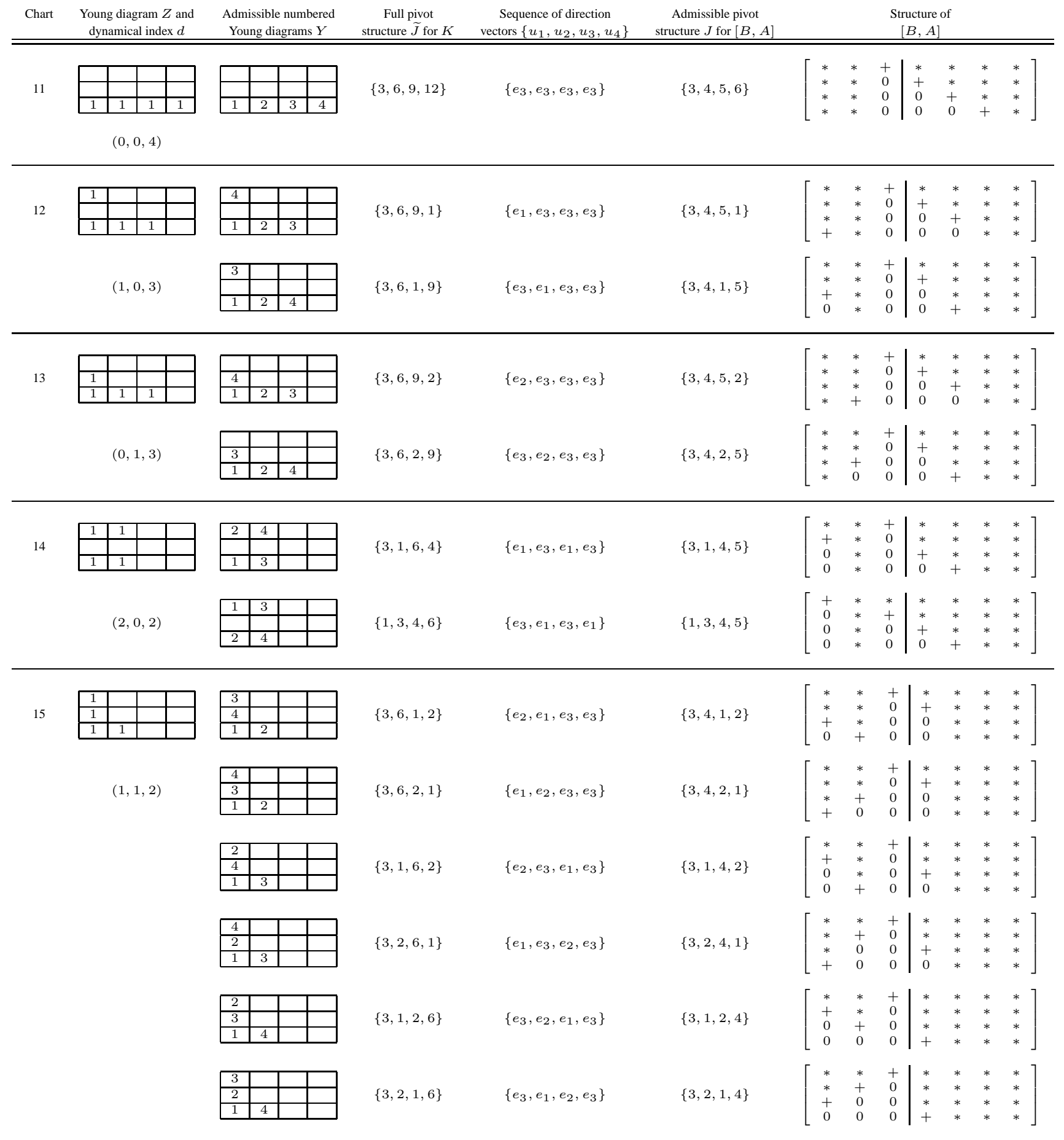

Table 3: Charts $11-15$ for $[B, A]$, for the case $m=3$ and $n=4$. 